\section{Arkivoc

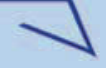

Archive for

Organic Chemistry
The Free Internet Journal

for Organic Chemistry
Paper

Arkivoc 2017, part ii, 118-137

\title{
Synthesis of substituted methylidenepyrimidobenzothiazolones as potential cytotoxic agents
}

\author{
Jakub Modranka, ${ }^{a}$ Anna Pietrzak ${ }^{\mathrm{b}}$, Wojciech M. Wolf ${ }^{\mathrm{b}}$ and Tomasz Janecki ${ }^{\mathrm{a}^{*}}$
}

a Institute of Organic Chemistry, Lodz University of Technology, Żeromskiego 116, 90-924 Łódź, Poland

${ }^{b}$ Institute of General and Ecological Chemistry, Lodz University of Technology, Żeromskiego 116, 90-924 Łódź,

Poland

E-mail: tomasz.janecki@p.lodz.pl

Dedicated to Prof. Jacek Młochowski on the occasion of his $80^{\text {th }}$ birthday

Received 05-23-2016

Accepted 07-14-2016

Published on line $08-23-2016$

\section{Abstract}

A range of biologically important substituted 3-methylidene-2,3-dihydro-4H-pyrimido[2,1- $b]$ [1,3]benzothiazol4-ones and 3-methylidene-3,4-dihydro-2H-pyrimido[2,1-b][1,3]benzothiazol-2-ones was synthesized applying Horner-Wadsworth-Emmons methodology for the introduction of exo-methylidene bond onto a heterocyclic ring. Crucial in this approach, phosphonates were prepared by the reaction of ethyl 2-diethoxyphosphoryl-3methoxyacrylate or ethyl 2-diethoxyphosphoryl-3-chloroacrylate with 2-aminobenzothiazoles, followed by addition of Grignard reagents to the obtained 3-diethoxyphosphoryl-4H-pyrimidobenzothiazol-4-ones or 3diethoxyphosphoryl-2H-pyrimido[2,1-b][1,3]benzothiazol-2-ones, respectively. Surprising, ambident behavior of 2-aminobenzothiazoles towards ethyl 2-diethoxyphosphoryl-3-methoxyacrylate and ethyl 2diethoxyphosphoryl-3-chloroacrylate is also discussed.

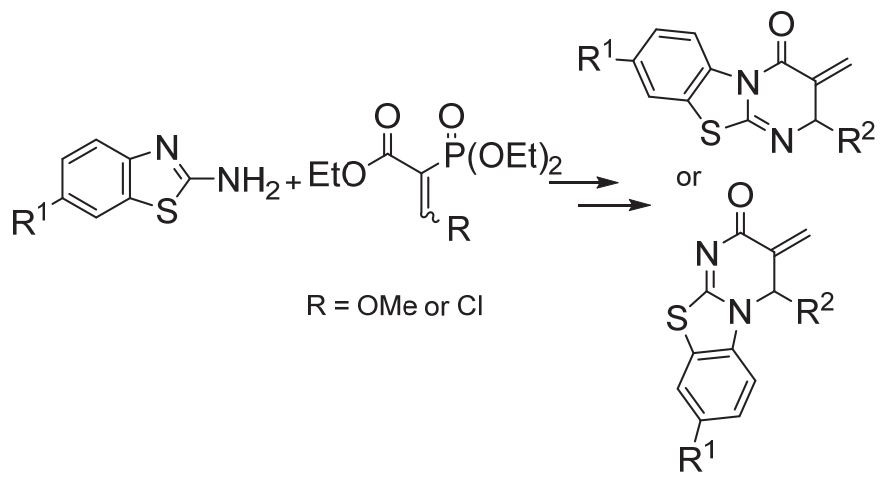

Keywords: Pyrimidobenzothiazolones, methylidenepyrimidobenzothiazolones, Michael addition, HornerWadsworth-Emmons olefination, phosphorylated azaheterocycles 


\section{Introduction}

Fused polyheterocycles, especially those containing nitrogen atoms, represent the core structural motif of a wide range of biologically active compounds. ${ }^{1}$ Not surprisingly, they are an important field of research and a very attractive target for the drug industry. One of such characteristic structural motifs are pyrimidobenzothiazolones, in which a pyrimidine ring is fused with another pharmacophorically active nucleus, benzothiazole, through a nitrogen atom. Synthesis and biological activity of both possible structural arrangements, $4 H$-pyrimido[2,1- $b][1,3]$ benzothiazol-4-ones 1 and $2 H$-pyrimido[2,1- $b][1,3]$ benzothiazol-2-ones 2 has been reported (Figure 1).

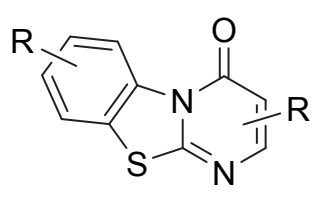

1

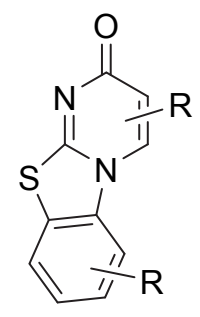

2

Figure 1. Structures of $4 H$-pyrimido[2,1-b][1,3]benzothiazol-4-ones $\quad 1$ and $2 H$-pyrimido[2,1b][1,3] benzothiazol-2-ones 2 .

Pyrimidobenzothiazol-4-ones 1 substituted at positions 2, 3 or in benzothiazole moiety were synthesized by condensation of 2-aminobenzothiazoles with diethyl malonates ${ }^{2}$ or ethyl acetoacetate ${ }^{3,4}$ or in the reaction of 2-aminobenzothiazoles with various Michael acceptors, such as diethyl alkoxymethylidenemalonates, $^{5-8}$ ethyl 2-cyano-3,3-bismethylthioacrylate, ${ }^{9,10}$ 2-cyano-3dimethylaminoacrylohydrazides $^{11}$ or dimethyl aminofumarate. ${ }^{6}$ Pyrimidobenzothiazol-4-ones 1 display interesting biological properties, for example anticancer, ${ }^{9-11}$ antimicrobial, ${ }^{2,4,9}$ antiallergic $^{6}$ or antifungal. ${ }^{3,4}$ Syntheses of $2 \mathrm{H}$-pyrimido[2,1-b][1,3]benzothiazol-2-ones 2 are usually less effective and were accomplished in a three-component reaction of a substituted benzaldehyde, malonate and 2-aminobenzothiazole ${ }^{12}$ or by heating 2-aminobenzothiazoles with propargylic acids, ${ }^{13}$ but-2-yn-1,4-diates ${ }^{14,15}$ or ethyl cyanoacetate. ${ }^{16}$ Another method is a microwave-promoted reaction of 2-aminobenzothiazoles with Baylis-Hillman acetates. ${ }^{17}$ Biological activity of $2 H$-pyrimido[2,1-b][1,3] benzothiazol-2-ones 2 is poorly recognized. It was reported that 4imino-3-aryldiazenyl-3,4-dihydro-2H-pyrimido[2,1- $b][1,3]$ benzothiazol-2-ones displayed antibacterial activity ${ }^{16}$ and several N'-substituted-4-carbohydrazide-3,4-dihydro-2H-pyrimido[2,1- $b][1,3]$ benzothiazol-2-ones have cytotoxic activity against kidney, lung, colon, prostate or breast cancer cell lines. ${ }^{14,15}$

Continuing our search for new heterocyclic frameworks with anticancer activity we decided to modify the structure of pyrimidobenzothiazolones by introducing exo-methylidene bond $\alpha$ to a carbonyl group. We assumed that such a modification might enhance the cytotoxic activity of the target methylidenepyrimidobenzothiazolones. Our reasoning was based on a well-established structure-activity relationship linking the strong cytotoxic activity displayed by a large group of natural and synthetic $\alpha$ alkylidenelactones and lactams with the presence of exo-alkylidene bond conjugated with a carbonyl group. $^{18,19}$ In this paper we present the synthesis of 3-methylidene-2,3-dihydro-4H-pyrimido[2,1b][1,3]benzothiazol-4-ones 11 and 3-methylidene-3,4-dihydro-2H-pyrimido[2,1-b][1,3]benzothiazol-2-ones 13 
using, well-recognized in our laboratory, Horner-Wadsworth-Emmons methodology for the introduction of the exo-methylidene double bond onto a heterocyclic ring. ${ }^{20}$

\section{Results and Discussion}

Following the success of our recent studies involving a new methodology which can be applied to the synthesis of diverse phosphorylated ortho-fused azaheterocycles ${ }^{21}$ we decided to further test the efficiency of 2-diethoxyphosphoryl-3-methoxyacrylate 4 in the preparation of 3-diethoxyphosphoryl-4H-pyrimido[2,1$b][1,3]$ benzothiazol-4-ones 6 , which are crucial intermediates in the present synthesis. To our delight, reacting methoxyacrylate 4 with 2-aminobenzothiazoles 3a-d in methanol at room temperature for 24 hours followed by the evaporation of methanol from the reaction mixture gave crude aminoacrylates 5a-d which were formed as mixtures of $E$ and $Z$ isomers. Their spectral data were in accordance with their structure. For example, in the ${ }^{31} \mathrm{P}$ NMR spectrum of crude 5 a only two signals from $E$ and $Z$ isomers were present $(\delta 20.10$ and 21.26 in 35/65 ratio, respectively). To simplify the procedure, crude aminoacrylates $\mathbf{5 a - d}$ were used in the next step. Heating them in Dowtherm $A$ at $250{ }^{\circ} \mathrm{C}$ for 30 minutes induced intramolecular cyclization and, after column chromatography, pure 3-diethoxyphosphorylpyrimidobenzothiazol-4-ones 6a-d were obtained in moderate to good yields (Scheme 1, Table 1). ${ }^{1} \mathrm{H},{ }^{13} \mathrm{C}$ and ${ }^{31} \mathrm{P}$ NMR spectra of 6 a-d were in full agreement with their structures.

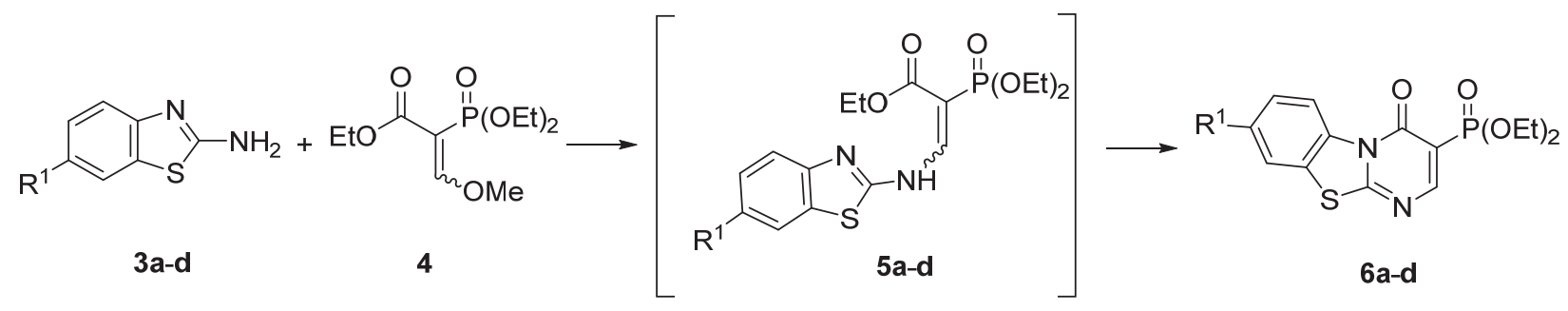

Scheme 1. Synthesis of 3-diethoxyphosphoryl-4H-pyrimidobenzothiazol-4-ones 6a-d. Reaction conditions: 1) methanol, $24 \mathrm{~h}, \mathrm{rt}$; 2) Dowtherm A, 30 min., $250{ }^{\circ} \mathrm{C}$.

Looking for milder reaction conditions for the synthesis of 6 , we decided to test the reaction of 2aminobenzothiazoles $\mathbf{3 a , b}, \mathbf{e}$ with 2-diethoxyphosphoryl-3-chloroacrylate $\mathbf{7}$. Chloroacrylate $\mathbf{7}$ has not been reported so far but turned out to be easily available by $\mathrm{SOCl}_{2}$ chlorination of ethyl 2-diethoxyphosphoryl-3hydroxyacrylate (see Experimental). Surprisingly, the reaction of $\mathbf{7}$ with 2-aminobenzothiazoles 3a,b,e in THF, in the presence of pyridine proceeded smoothly at room temperature giving proiducts isomeric to 6 - 3diethoxyphosphoryl-2H-pyrimido[2,1-b][1,3]benzothiazol-2-ones 9a,b,e, in good yields (Scheme 2, Table 1). In this reaction we were unable to isolate the intermediate substitution products $\mathbf{8 a}, \mathbf{b}, \mathbf{e}$. The NMR spectra of $\mathbf{9 a , b , e ~ w e r e ~ i n ~ a c c o r d a n c e ~ w i t h ~ t h e i r ~ s t r u c t u r e s . ~ F u r t h e r m o r e , ~ t h e ~ t w o ~ r e g i o i s o m e r s ~} \mathbf{6 a}$ and $\mathbf{9 a}$ were selected for X-ray single crystal analysis to explicitly confirm their structures. The crystal structure of 9a is reported herein. Crystal data for $6 a$ have been already deposited in the Cambridge Structural Database ${ }^{22}$ by us as part of a publication on new crystal packing motifs. Views of molecules 6a and 9a as determined in the crystalline state are presented in Figure 2. Surprisingly, conformation around the exocyclic P1-C1 bond differs significantly in these two regioisomers. It can be conveniently defined by the 04-P1-C1-C10 torsion angle which adopts values $-176.20(13)^{\circ}$ (anti) and $-65.23(13)^{\circ}$ (gauche) in $6 \mathbf{6}$ and $9 \mathrm{a}$, respectively. The gauche 
conformation as in $9 \mathrm{a}$ is additionally stabilized by the hydrogen bond between a phosphoryl oxygen atom and the water molecule which was localized in the crystal.

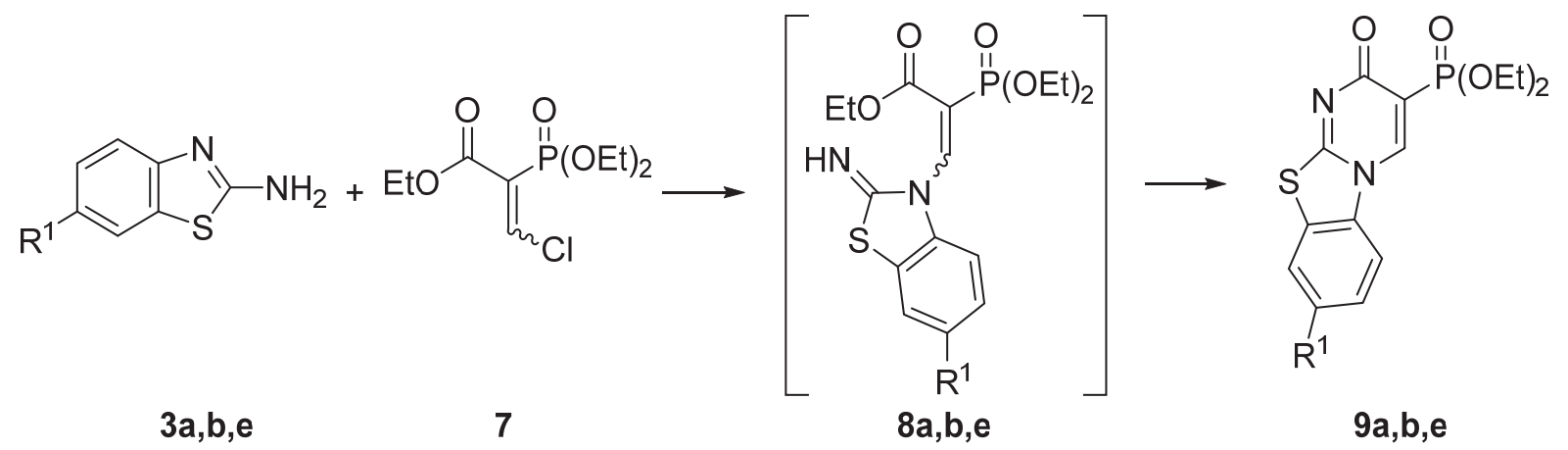

Scheme 2. Synthesis of 3-diethoxyphosphoryl-2H-pyrimido[2,1-b][1,3]benzothiazol-2-ones 9a,b,e. Reaction conditions: THF, pyridine, $24 \mathrm{~h}, \mathrm{rt}$.

Table 1. 3-Diethoxyphosphoryl-4H-pyrimido[2,1-b][1,3]benzothiazol-4-ones 6a-d and 3-diethoxyphosphoryl$2 \mathrm{H}$-pyrimido[2,1-b][1,3]benzothiazol-2-ones 9 a, b,e obtained

\begin{tabular}{cccc}
\hline Compound & $\mathrm{R}^{1}$ & $\begin{array}{c}\mathbf{6} \\
\text { Yield (\%) }\end{array}$ & $\begin{array}{c}\mathbf{9} \\
\text { Yield (\%) }\end{array}$ \\
\hline a & $\mathrm{H}$ & 89 & 96 \\
b & $\mathrm{Me}$ & 48 & 58 \\
c & $\mathrm{Cl}$ & 63 & - \\
d & $\mathrm{NO}_{2}$ & 71 & - \\
e & $\mathrm{OMe}^{\mathrm{M} e}$ & - & 76 \\
\hline
\end{tabular}

${ }^{\text {a }}$ Yield of isolated, purified product, based on 3.

The unexpected formation of pyrimidobenzothiazol-2-ones 9 can be rationalized assuming that the addition of 2-aminobenzothiazoles 3 to chloroacrylate $\mathbf{7}$ proceeds via the imine nitrogen atom and, after the elimination of chloride, substitution products 8 are formed, which undergo spontaneous intramolecular cyclization to yield $\mathbf{9}$. It is worth mentioning, that previously reported additions of 2-aminobenzothiazoles to 2 alkoxyalkylidenemalonates ${ }^{5-8}$ or ethyl 2-cyano-3,3-bis(methylthio)acrylate ${ }^{9,10}$ proceeded always via the amine nitrogen, yielding 3-alkoxycarbonyl or 3-cyanopyrimidobenzothiazol-4-ones, respectively. In turn, addition of 2-aminobenzothiazole $3 a$ to alkyl 2-arylidenemalonates proceeds via the imine nitrogen atom. ${ }^{12}$ Reactions of 2-aminobenzothiazoles with 2-chloromethylidenemalonates have not been reported. To shed more light on the observed phenomenon, we performed the reaction of 2-diethoxyphosphoryl-3-methoxyacrylate 4 with 2aminobenzothiazole $\mathbf{3 a}$ in THF, in the presence of pyridine and it turned out that the substitution product $\mathbf{5 a}$ was formed exclusively. Therefore the different regioselectivity noticed for the substrates $\mathbf{4}$ and $\mathbf{7}$ is caused by the different substrate structure rather than the different reaction conditions. However, full understanding of our observation certainly needs further investigation. Nevertheless, the fully regioselective, ambident reactivity of 2-aminobenzothizoles $\mathbf{3}$ towards $\mathbf{4}$ and $\mathbf{7}$ gives obvious synthetic advantages and the potential of this phenomenon is currently being tested in our laboratory. 


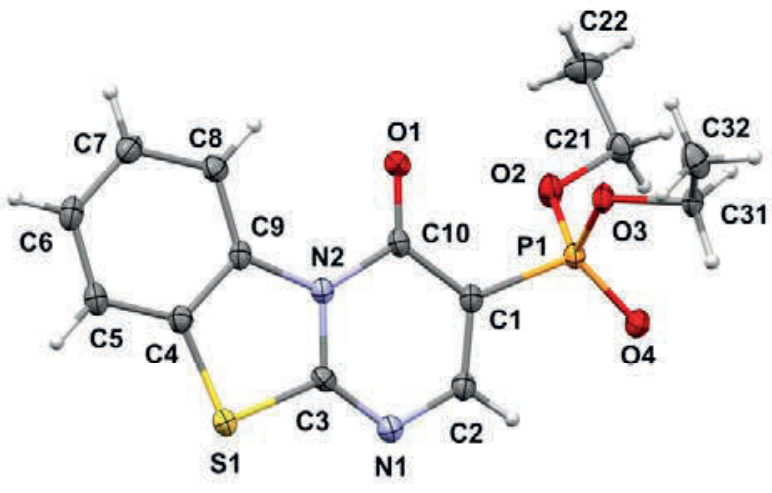

$6 a$

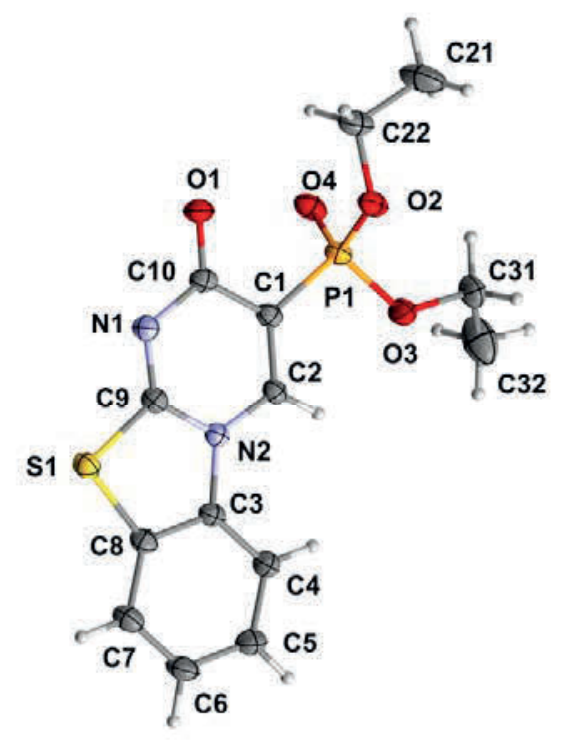

9a

Figure 2. A view of molecules $\mathbf{6 a}$ and $\mathbf{9 a}$ (hydrogen labels and disordered water molecule as in 9a are omitted for clarity). Displacement ellipsoids are drawn at the 50\% probability level. Selected geometric parameters; 6a: P1-C1 1.7813(16)Å, C3-S1-C4 90.35(8) ${ }^{\circ}$, O4-P1-C1 112.99(7) ${ }^{\circ}$, C3-N1-C2 115.07(14) ${ }^{\circ}$, C3-N2-C10 121.11(13) , C2-C1-C10 121.08(14) ${ }^{\circ}$, O4-P1-C1-C10 -176.20(13) ; 9a: P1-C1 1.7801(15)Å, C9-S1-C8 90.62(7) ${ }^{\circ}$, O4-P1-C1 $114.39(7)^{\circ}, \mathrm{C} 9-\mathrm{N} 1-\mathrm{C} 10118.35(13)^{\circ}, \mathrm{C} 2-\mathrm{C} 1-\mathrm{C} 10120.17(14)^{\circ}, \mathrm{C} 2-\mathrm{N} 2-\mathrm{C} 9118.77(13)^{\circ}$, O4-P1-C1-C10 -65.23(13) ${ }^{\circ}$.

The synthesised 3-diethoxyphosphorylpyrimidobenzothiazol-4-ones 6a-d and 3diethoxyphosphorylpyrimidobenzothiazol-2-ones 9a,b,e were next used as Michael acceptors in reactions with various Grignard reagents. Additions took place effectively in the presence of Cul and after a standard work-up and purification by column chromatography, 2-substituted 3-diethoxyphosphoryl-2,3-dihydro-4Hpyrimido[2,1-b][1,3]benzothiazol-4-ones $10 \mathrm{a}, \mathbf{d}, \mathbf{e}, \mathrm{i}-\mathrm{I}$ and 3-diethoxyphosphoryl-3,4-dihydro-2H-pyrimido[2,1b][1,3]benzothiazol-2-ones $\mathbf{1 2 a - j , m}$ were obtained in good to excellent yields (Scheme 3, Table 2). Only additions to benzothiazol-4-one $\mathbf{6 d}$ were ineffective and always gave a complex mixture of products. Benzothiazol-4-ones $\mathbf{1 0 e}, \mathbf{j}, \mathbf{I}$ substituted with a phenyl group were obtained as single trans isomers and benzothiazol-4-ones $\mathbf{1 0 a}, \mathbf{d}, \mathbf{i}, \mathbf{k}$ as a mixture of trans and cis isomers in a ratio given in Table 2 . In turn, benzothiazol-2-ones $\mathbf{1 2 a - j , m}$ were all formed as single trans isomers. Formation of trans-benzothiazolones $\mathbf{1 0}$ and $\mathbf{1 2}$ as major or single stereoisomers is in accordance with a well-established observation, that in this type of Michael addition, thermodynamic control is usually observed. ${ }^{23,24}$ Analysis of ${ }^{1} \mathrm{H},{ }^{13} \mathrm{C}$ and ${ }^{31} \mathrm{P}$ NMR spectra fully confirmed the structures of benzothiazolones 10 and 12 and their stereochemistry. Diagnostic for a trans diaxial arrangement of diethoxyphosphoryl group and $\mathrm{R}^{2}$ substituent were coupling constants ${ }^{3} \mathrm{~J}_{\mathrm{H} 3-\mathrm{H} 2}=0.9-1.2$ $\mathrm{Hz}$ in trans-benzothiazol-4-ones 10 and ${ }^{3} \mathrm{~J}_{\mathrm{H} 3-\mathrm{H} 4}=0.0-0.8 \mathrm{~Hz}$ in trans-benzothiazol-2-ones 12 . Corresponding coupling constants ${ }^{3} \mathrm{~J}_{\mathrm{H} 3-\mathrm{H} 2}$ for cis-benzothiazol-4-ones $\mathbf{1 0 a}, \mathbf{d}, \mathbf{i}, \mathbf{k}$ were in the range of 5.5-5.7 $\mathrm{Hz}$. Also, coupling constants ${ }^{3} J_{R 2-p}$ in all trans-benzothiazolones 10 and 12 were in the range of $17.0-18.7 \mathrm{~Hz}$. Unfortunately, we were unable to determine the ${ }^{3} J_{R 2-P}$ coupling constants from ${ }^{13} \mathrm{C} N M R$ spectra of cis-benzothiazol-4-ones $10 \mathbf{a}, \mathbf{d}, \mathbf{i}, \mathbf{k}$, due to small amount of these isomers in the mixture. These data are in full agreement with the corresponding coupling constants observed for trans-4-alkyl-3-diethoxyphosphorylchroman-2-ones with a diaxial arrangement of alkyl and diethoxyphosphoryl group. ${ }^{25,26}$ 
In the final step of our synthesis, pyrimidobenzothiazolones 10 and 12 were employed in HornerWadsworth-Emmons olefinations with formaldehyde. Reaction of pyrimidobenzothiazol-4-ones 10a,d,e,i-I with an excess of paraformaldehyde proceeded smoothly in the presence of $\mathrm{NaH}$ as a base. Crude 3methylidene-2,3-dihydro-4H-pyrimido[2,1-b][1,3]benzothiazol-4-ones $\mathbf{1 1 a , d , e , i , j ~ w e r e ~ p u r i f i e d ~ b y ~ c o l u m n ~}$ chromatography to give the target compounds in good to moderate yields (Scheme 3, Table 2). Disappointingly, methylidenepyrimidobenzothiazolones $\mathbf{1 1 k} \mathbf{l} \mathbf{l}$ were very unstable and decomposed during attempted purification by column chromatography. Decomposition was noticable also during the storage of the crude compounds in a refrigerator for several hours. The ${ }^{1} \mathrm{H}$ NMR spectra of $11 \mathbf{k}, \mathrm{I}$ given in the experimental section were therefore registered immediately after the reaction, using crude products. Slow decomposition was also observed for the remaining methylidenepyrimidobenzothiazolones $\mathbf{1 1}$, even if they were kept in the refrigerator.

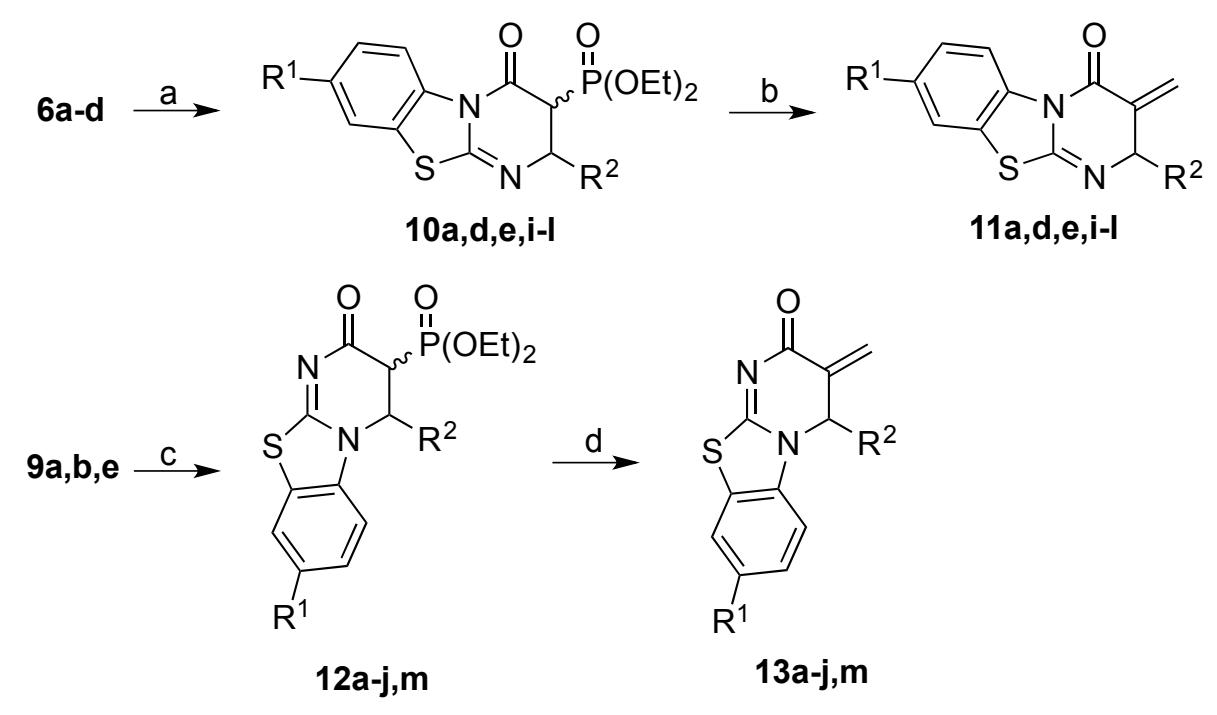

Scheme 3. Synthesis of methylidenepyrimidobenzothiazolones 11a,d,e,i-I and 13a-j,m. Reaction conditions: (a) $\mathrm{R}^{2} \mathrm{MgCl}$, cat. Cul, THF, $-78{ }^{\circ} \mathrm{C}$ to rt; b) $\mathrm{NaH},\left(\mathrm{CH}_{2} \mathrm{O}\right)_{n}$, THF, rt, $4 \mathrm{~h}$; c) R ${ }^{2} \mathrm{MgCl}, \mathrm{THF}, 0{ }^{\circ} \mathrm{C}$ to rt; d) $\mathrm{K}_{2} \mathrm{CO}_{2},\left(\mathrm{CH}_{2} \mathrm{O}\right)_{n}$, THF, rt, 24 h.

Table 2. Substituted 3-diethoxyphosphorylpyrimidobenzothiazolones 10a,d,e,i-I and 12a-j,m and methylidenepyrimidobenzothiazolones $11 \mathrm{a}, \mathrm{d}, \mathrm{e}, \mathrm{i}-\mathrm{I}$ and $13 \mathrm{a}-\mathrm{j}, \mathrm{m}$ obtained

\begin{tabular}{|c|c|c|c|c|c|c|c|}
\hline \multirow[b]{2}{*}{ Compound } & \multirow[b]{2}{*}{$\mathrm{R}^{1}$} & \multirow[b]{2}{*}{$\mathrm{R}^{2}$} & \multicolumn{2}{|c|}{10} & \multirow[b]{2}{*}{$\begin{array}{c}11 \\
\text { Yield }^{\mathrm{b}}(\%)\end{array}$} & \multirow[b]{2}{*}{$\begin{array}{c}12 \\
\text { Yield }^{\mathrm{b}}(\%)\end{array}$} & \multirow[b]{2}{*}{$\begin{array}{c}13 \\
\text { Yield }^{\mathrm{b}}(\%)\end{array}$} \\
\hline & & & $\begin{array}{c}\text { trans/cis } \\
\text { ratio }^{a}\end{array}$ & $\begin{array}{c}\text { Yield }^{\mathrm{b}} \\
(\%)\end{array}$ & & & \\
\hline$a$ & $\mathrm{H}$ & $\mathrm{Me}$ & $88 / 12$ & 80 & 51 & 73 & 76 \\
\hline b & $\mathrm{H}$ & Et & - & - & - & 68 & 82 \\
\hline C & $\mathrm{H}$ & $i-\operatorname{Pr}$ & - & - & - & 43 & 32 \\
\hline d & $\mathrm{H}$ & $n-\mathrm{Bu}$ & $94 / 6$ & 75 & 34 & 72 & 87 \\
\hline e & $\mathrm{H}$ & $\mathrm{Ph}$ & $>99 / 1$ & 93 & 69 & 68 & 61 \\
\hline$f$ & $\mathrm{Me}$ & $\mathrm{Me}$ & - & - & - & 71 & 82 \\
\hline g & Me & Et & - & - & - & 85 & 84 \\
\hline h & Me & $i-\operatorname{Pr}$ & - & - & - & 78 & 39 \\
\hline
\end{tabular}


Table 2. Continued

\begin{tabular}{|c|c|c|c|c|c|c|c|}
\hline \multirow[b]{2}{*}{ Compound } & \multirow[b]{2}{*}{$\mathrm{R}^{1}$} & \multirow[b]{2}{*}{$\mathrm{R}^{2}$} & \multicolumn{2}{|c|}{10} & \multirow[b]{2}{*}{$\begin{array}{c}11 \\
\text { Yield }^{\mathrm{b}}(\%)\end{array}$} & \multirow[b]{2}{*}{$\begin{array}{c}12 \\
\text { Yield }^{\mathrm{b}}(\%)\end{array}$} & \multirow[b]{2}{*}{$\begin{array}{c}13 \\
\text { Yield }^{\mathrm{b}}(\%)\end{array}$} \\
\hline & & & $\begin{array}{c}\text { trans/cis } \\
\text { ratio }^{a}\end{array}$ & $\begin{array}{c}\text { Yield }^{b} \\
(\%)\end{array}$ & & & \\
\hline $\mathbf{i}$ & $\mathrm{Me}$ & $n$-Bu & $93 / 7$ & 79 & 76 & 74 & 87 \\
\hline $\mathbf{j}$ & Me & $\mathrm{Ph}$ & $>99 / 1$ & 83 & 52 & 79 & 71 \\
\hline k & $\mathrm{Cl}$ & $n-\mathrm{Bu}$ & $94 / 6$ & 95 & $-^{c}$ & - & - \\
\hline I & $\mathrm{Cl}$ & $\mathrm{Ph}$ & $>99 / 1$ & 85 & $-^{c}$ & - & - \\
\hline$m$ & OMe & $n$-Bu & - & - & - & 89 & 76 \\
\hline
\end{tabular}

${ }^{\mathrm{a}}$ Ratio determined from ${ }^{31} \mathrm{P}$ NMR spectra of the crude compounds.

${ }^{\mathrm{b}}$ Yield of isolated, purified product, based on 6, 10, 9 or 12, respectively.

${ }^{\mathrm{c}}$ Yield was not determined due to fast decomposition of the obtained compounds.

In turn, synthesis of 3-methylidene-3,4-dihydro-2H-pyrimido[2,1-b][1,3]benzothiazol-2-ones 13a-j, m was accomplished when pyrimidobenzothiazol-2-ones $\mathbf{1 2} \mathbf{a}-\mathbf{j}, \mathbf{m}$ were treated with paraformaldehyde in the presence of $\mathrm{K}_{2} \mathrm{CO}_{3}$ as a base. Purification of the crude products by column chromatography gave methylidenepyrimidobenzothiazolones $\mathbf{1 3 a - j , m}$ in good to moderate yields (Scheme 3, Table 2). In contrast to the methylidenepyrimidobenzothiazol-4-ones 11, methylidenepyrimidobenzothiazol-2-ones 13 were stable at room temperature for at least several weeks.

In conclusion, we have performed an efficient synthesis of new, biologically important methylidenepyrimidobenzothiazolones $\mathbf{1 1}$ and $\mathbf{1 3}$ using Horner-Wadsworth-Emmons methodology. It is worth stressing that key intermediates $\mathbf{6}$ and $\mathbf{9}$ were effectively obtained due to the unexpected discovery of the ambient and fully regioselective behavior of 2-aminobenzothiazoles 3 toward methoxyacrylate 4 and chloroacrylate 7. The structure of both regioisomers $6 \mathrm{a}$ and $9 \mathrm{a}$ were unequivocally determined by $\mathrm{X}$-ray structure analysis. Currently, target methylidenepyrimidobenzothiazolones $\mathbf{1 3}$ are being assessed for their cytotoxicity and preliminary tests show that they are highly active. These, very interesting biological results will be published shortly.

\section{Experimental Section}

General. NMR spectra were recorded on a Bruker DPX 250 or Bruker Avance II instrument at $250.13 \mathrm{MHz}$ or $700 \mathrm{MHz}$ for ${ }^{1} \mathrm{H}, 62.9 \mathrm{MHz}$ or $176 \mathrm{MHz}$ for ${ }^{13} \mathrm{C}$, and $101.3 \mathrm{MHz}$ or $283 \mathrm{MHz}$ for ${ }^{31} \mathrm{P} \mathrm{NMR}$ using tetramethylsilane as internal and $85 \% \mathrm{H}_{3} \mathrm{PO}_{4}$ as external standard. ${ }^{31} \mathrm{P} \mathrm{NMR}$ spectra were recorded using broadband proton decoupling. IR spectra were recorded on a Bruker Alpha ATR spectrophotometer. Melting points were determined in open capillaries and are uncorrected. Column chromatography was performed on Aldrich ${ }^{\circledR}$ silica gel 60 (230-400 mesh). Thin-layer chromatography was performed with precoated TLC sheets of silica gel 60 $\mathrm{F}_{254}\left(\right.$ Aldrich $\left.^{\circledR}\right)$. The purity of tested compounds was determined by combustion elemental analyses (CHN, elemental analyzer EuroVector 3018, Elementar Analysensysteme $\mathrm{GmbH}$ ). Reagents and starting materials were purchased from commercial vendors and used without further purification. All organic solvents were dried over appropriate drying agents and distilled prior to use. Standard syringe techniques were used for transferring dry solvents. The crystal data were collected on a Bruker Smart APEX2 diffractometer at $100 \mathrm{~K}$ 


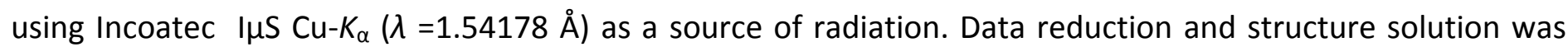
performed with the Bruker APEX2 Suite of programs. ${ }^{27}$ The ShelXle/XL were further applied for structure refinement and visualization. The CCDC Mercury and Mogul ${ }^{28}$ programs were used for molecular geometry and crystal packing examinations.

Ethyl 3-chloro-2-(diethoxyphosphoryl)acrylate (7). To a mixture of triethyl phosphonoacetate (10.5 $\mathrm{mL}, 52$ $\mathrm{mmol})$ and ethyl formate $(14.1 \mathrm{~mL}, 170 \mathrm{mmol})$ in EtOH $(20 \mathrm{~mL}) \mathrm{NaOEt}$ in EtOH $(15 \%, 100 \mathrm{mmol})$ was added. The reaction was stirred at ambient temperature for $3 \mathrm{~d}$. The mixture was concentrated in vacuo, $\mathrm{CH}_{2} \mathrm{Cl}_{2}(100$ $\mathrm{mL}$ ) was added, and the mixture was acidified to $\mathrm{pH}$ ca. 1.5 with $10 \%$ aq $\mathrm{HCl}$ solution. The organic layer was separated, dried over $\mathrm{Na}_{2} \mathrm{SO}_{4}$ and concentrated under reduced pressure. The crude ethyl 2(diethoxyphosphoryl)-3-hydroxyacrylate was dissolved in toluene $(75 \mathrm{~mL})$, then $\mathrm{SOCl}_{2}(4 \mathrm{~mL}, 55 \mathrm{mmol})$ followed by catalytic amount of DMF $(0.1 \mathrm{~mL})$ were added. The mixture was heated under reflux for $4 \mathrm{~h}$. The solvent was evaporated, and the crude product was distilled under reduced pressure to afford pale yellow product 7 (87\%, bp 100-110 $\left.{ }^{\circ} \mathrm{C} / 0.4 \mathrm{mbar}\right)$ as a mixture of two isomers $E / Z=20 / 1 . \mathrm{IR} \mathrm{v}\left(\mathrm{cm}^{-1}\right): 2984(\mathrm{w}), 1727$ (m), 1211 (s), 1011 (vs), 974 (vs); ${ }^{1} \mathrm{H}$ NMR (250 MHz, CDCl $) \delta 7.80$ (d, J 35.0 Hz, 1H, minor, H-3), 7.41 (d, J 14.4 $\mathrm{Hz}, 1 \mathrm{H}$, major, $\mathrm{H}-3), 4.32\left(\mathrm{q}, J 7.1 \mathrm{~Hz}, 2 \mathrm{H}\right.$, major and minor, $\left.\mathrm{CH}_{3} \mathrm{CH}_{2} \mathrm{OC}(\mathrm{O})\right)$ ), $4.07-4.26(\mathrm{~m}, 4 \mathrm{H}$, major and minor, $\left.\left(\mathrm{CH}_{3} \mathrm{CH}_{2} \mathrm{O}\right)_{2} \mathrm{P}(\mathrm{O})\right)$, 1.30-1.36 (m, 9H, major and minor, $\mathrm{CH}_{3} \mathrm{CH}_{2} \mathrm{OC}(\mathrm{O})$, $\left.\left(\mathrm{CH}_{3} \mathrm{CH}_{2} \mathrm{O}\right)_{2} \mathrm{P}(\mathrm{O})\right)$; ${ }^{13} \mathrm{C} \mathrm{NMR} \mathrm{(63}$ $\mathrm{MHz}_{\mathrm{CDCl}}$, major) $\delta 162.5$ (d, J $9.7 \mathrm{~Hz}, \mathrm{C}-1$ ), 140.3 (d, J $18.1 \mathrm{~Hz}, \mathrm{C}-3$ ), 128.6 (d, J $175.4 \mathrm{~Hz}, \mathrm{C}-2$ ), 63.2 (d, J 5.4 $\mathrm{Hz}, \mathrm{CH}_{3} \mathrm{CH}_{2} \mathrm{OP}(\mathrm{O})$ ), 61.9 (s, $\mathrm{CH}_{3} \mathrm{CH}_{2} \mathrm{OC}(\mathrm{O})$ ), 16.2 (d, J $6.6 \mathrm{~Hz},\left(\mathrm{CH}_{3} \mathrm{CH}_{2} \mathrm{O}\right)_{2} \mathrm{P}(\mathrm{O})$ ), 14.0 (s, $\mathrm{CH}_{3} \mathrm{CH}_{2} \mathrm{OC}(\mathrm{O})$ ); ${ }^{31} \mathrm{P} \mathrm{NMR}$ (101 MHz, $\mathrm{CDCl}_{3}$ ) $\delta$ 9,60 (major), 7,96 (minor). Anal. Calcd for $\mathrm{C}_{9} \mathrm{H}_{16} \mathrm{ClO}_{5} \mathrm{P}$ (270.65): C, 39.94; $\mathrm{H}, 5.94 \%$. Found: C, $39.87 ; \mathrm{H}, 5.98 \%$.

General procedure for the synthesis of diethyl (4-oxo-4H-benzothiazolopyrimidin-3-yl)phosphonates 6a-c,e. To a solution of 2-aminobenzotriazole 3a-c,e $(10.0 \mathrm{mmol})$ in $\mathrm{MeOH}(50 \mathrm{~mL})$ 2-diethoxyphosphoryl-3methoxyacrylate (4) $(2.66 \mathrm{~g}, 10.0 \mathrm{mmol}$ ) was added and the mixture was stirred for $24 \mathrm{~h}$. Next, the $\mathrm{MeOH}$ was evaporated and Dowtherm A (150 mL) was added. The mixture was heated under reflux for 30 minutes. After cooling, the reaction mixture was applied to a silica gel column. The column was washed in turn with hexane $(150 \mathrm{~mL})$, EtOAc $(150 \mathrm{~mL})$ and EtOH $(150 \mathrm{~mL})$. The EtOH fraction was evaporated and the residue purified by column chromatography (eluent: EtOAc-MeOH, 10:1).

Diethyl (4-oxo-4H-benzo[4,5]thiazolo[3,2-a]pyrimidin-3-yl)phosphonate (6a). (89\%); yellow crystals; mp 70$73{ }^{\circ} \mathrm{C} ; \mathrm{IR} \mathrm{v}\left(\mathrm{cm}^{-1}\right)$ : $2978(\mathrm{w}), 1691(\mathrm{~m}), 1486$ (s), 1243 (s), 1013 (vs), 956 (vs); ${ }^{1} \mathrm{H}$ NMR (700 MHz, CDCl 3 ) $\delta 9.18-$ $9.10(\mathrm{~m}, 1 \mathrm{H}, \mathrm{H}-\mathrm{Ar}), 8.57(\mathrm{~d}, J 9.6 \mathrm{~Hz}, 1 \mathrm{H}, H-2), 7.79-7.71(\mathrm{~m}, 1 \mathrm{H}, \mathrm{H}-\mathrm{Ar}), 7.62-7.51(\mathrm{~m}, 2 \mathrm{H}, H-\mathrm{Ar}), 4.38-4.15$ (m, 4H, CH $\mathrm{CH}_{2} \mathrm{OP}, 1.39$ (t, J $\left.7.0 \mathrm{~Hz}, 6 \mathrm{H}, \mathrm{CH}_{3} \mathrm{CH}_{2} \mathrm{O}\right) ;{ }^{13} \mathrm{C} \mathrm{NMR}\left(176 \mathrm{MHz}, \mathrm{CDCl}_{3}\right) \delta 166.5$ (s, C-Ar), $159.3(\mathrm{~d}, J 9.5 \mathrm{~Hz}$, C-3), 158.9 (d, J 12.7 Hz, C(O)), 135.7 (s, C-Ar), 127.7 (s, CH-Ar), 127.5 (s, CH-Ar), 124.1 (s, C-Ar), 121.9 (s, CHAr), 120.4 (s, CH-Ar), 108.8 (d, J 197.7 Hz, C-2), 62.8 (d, J 3.1 Hz, CH $2 \mathrm{OP}$ ), 62.8 (d, J 4.2 Hz, CH ${ }_{2} \mathrm{OP}$ ), 16.4 (d, J 6.3 $\mathrm{Hz}, \mathrm{CH}_{3} \mathrm{CH}_{2} \mathrm{OP}$ ); ${ }^{31} \mathrm{P} \mathrm{NMR}\left(283 \mathrm{MHz}, \mathrm{CDCl}_{3}\right) \delta$ 13.91. Anal. Calcd for $\mathrm{C}_{14} \mathrm{H}_{15} \mathrm{~N}_{2} \mathrm{O}_{4} \mathrm{PS}$ (338.32): C, 49.70; $\mathrm{H}, 4.47 ; \mathrm{N}$ 8.28\%. Found: C, 49.59; H, 4.50; N 8.26\% CCDC 1478184 contains the crystallographic data of 6a. These data can be obtained free of charge from The Cambridge Crystallographic Data Centre via www.ccdc.cam.ac.uk/data request/cif.

Diethyl (8-methyl-4-oxo-4H-benzo[4,5]thiazolo[3,2-a]pyrimidin-3-yl)phosphonate (6b). (48\%); yellow

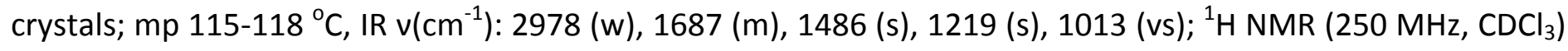
$\delta 8.97$ (d, J $8.7 \mathrm{~Hz}, 1 \mathrm{H}, H-\mathrm{Ar}$ ), 8.53 (d, J $9.6 \mathrm{~Hz}, 1 \mathrm{H}, H-2), 7.53$ (d, J $1.4 \mathrm{~Hz}, 1 \mathrm{H}, H-\mathrm{Ar}$ ), 7.37 (dd, J 8.7, $1.4 \mathrm{~Hz}, 1 \mathrm{H}$, H-Ar), $4.40-4.15\left(\mathrm{~m}, 4 \mathrm{H}, \mathrm{CH}_{2} \mathrm{OP}\right), 2.50\left(\mathrm{~s}, 3 \mathrm{H}, \mathrm{CH}_{3}\right), 1.23\left(\mathrm{t}, J 7.0 \mathrm{~Hz}, 6 \mathrm{H}, \mathrm{CH}_{3} \mathrm{CH}_{2} \mathrm{O}\right) ;{ }^{13} \mathrm{C} \mathrm{NMR}\left(63 \mathrm{MHz}^{\mathrm{CDCl}}\right)_{3} \delta$ 
166.3 (s, C-Ar), 159.1 (d, J $11.3 \mathrm{~Hz}, C-3$ ), 158.7 (d, J $12.7 \mathrm{~Hz}, C(\mathrm{O})$ ), 138.1 (s, C-Ar), 133.4 (s, C-Ar), 128.5 (s, CH$\mathrm{Ar}$ ), 124.0 (s, C-Ar), 121.7 (s, CH-Ar), 120.0 (s, CH-Ar), 108.4 (d, J $197.6 \mathrm{~Hz}, \mathrm{C}-2$ ), 62.7 (d, J 5.8 Hz, CH $\mathrm{H}_{2} \mathrm{OP}$ ), 21.3 (s, $\left.\mathrm{CH}_{3}\right), 16.3\left(\mathrm{~d}, J 6.4 \mathrm{~Hz}, \mathrm{CH}_{3} \mathrm{CH}_{2} \mathrm{OP}\right) ;{ }^{31} \mathrm{P} \mathrm{NMR}\left(101 \mathrm{MHz}, \mathrm{CDCl}_{3}\right) \delta$ 14.94. Anal. Calcd for $\mathrm{C}_{15} \mathrm{H}_{17} \mathrm{~N}_{2} \mathrm{O}_{4} \mathrm{PS}$ (352.34): C, 51.13; H, 4.86; N 7.95\%. Found: C, 51.00; H, 4.85; N 7.99\%.

Diethyl (8-chloro-4-oxo-4H-benzo[4,5]thiazolo[3,2-a]pyrimidin-3-yl)phosphonate (6c). (63\%); yellow crystals; $\mathrm{mp} 145-147{ }^{\circ} \mathrm{C}$, IR v(cm $\left.{ }^{-1}\right): 2981(\mathrm{w}), 1690(\mathrm{~m}), 1485$ (s), 1250 (s), $1026(\mathrm{vs}) ;{ }^{1} \mathrm{H} \mathrm{NMR}\left(700 \mathrm{MHz}, \mathrm{CDCl}_{3}\right) \delta 9.07$ (d, J $9.0 \mathrm{~Hz}, 1 \mathrm{H}, H-\mathrm{Ar}$ ), 8.56 (d, J $9.6 \mathrm{~Hz}, 1 \mathrm{H}, H-2), 7.74$ (d, J $2.1 \mathrm{~Hz}, 1 \mathrm{H}, H-\mathrm{Ar}$ ), 7.55 (dd, J 9.0, 2.1 Hz, 1H, H-Ar), $4.34-4.22\left(\mathrm{~m}, 4 \mathrm{H}, \mathrm{CH}_{2} \mathrm{OP}\right), 1.39\left(\mathrm{t}, J 7.1 \mathrm{~Hz}, 6 \mathrm{H}, \mathrm{CH}_{3} \mathrm{CH}_{2} \mathrm{O}\right) ;{ }^{13} \mathrm{C} \mathrm{NMR}\left(176 \mathrm{MHz}^{\mathrm{C} C D C l}\right)_{3} \delta 166.0(\mathrm{~s}, \mathrm{C}-\mathrm{Ar}), 159.3$ (d, J $11.3 \mathrm{~Hz}, C-3$ ), 158.7 (d, J $12.7 \mathrm{~Hz}, C(\mathrm{O})$ ), 134.2 (s, C-Cl), 133.5 (s, C-Ar), 127.9 (s, CH-Ar), 125.7 (s, CH-Ar), 121.8 (s, C-Ar), 121.2 (s, CH-Ar), 109.3 (d, J $197.3 \mathrm{~Hz}, C-2$ ), 62.9 (d, J $5.7 \mathrm{~Hz}, \mathrm{CH}_{2} \mathrm{OP}$ ), 16.4 (d, J $6.3 \mathrm{~Hz}$, $\mathrm{CH}_{3} \mathrm{CH}_{2} \mathrm{OP}$ ); ${ }^{31} \mathrm{P}$ NMR (283 MHz, $\left.\mathrm{CDCl}_{3}\right) \delta$ 13.37. Anal. Calcd for $\mathrm{C}_{14} \mathrm{H}_{14} \mathrm{ClN}_{2} \mathrm{O}_{4} \mathrm{PS}$ (372.76): $\mathrm{C}, 45.11 ; \mathrm{H}, 3.79 ; \mathrm{N}$ 7.52\%. Found: C, 44.98; $\mathrm{H}, 3.82 ; \mathrm{N} 7.51 \%$.

Diethyl (8-nitro-4-oxo-4H-benzo[4,5]thiazolo[3,2-a]pyrimidin-3-yl)phosphonate (6e). (71\%); yellow crystals; $\mathrm{mp} 125-127{ }^{\circ} \mathrm{C}$, IR v(cm $\left.{ }^{-1}\right): 2984(\mathrm{w}), 1606$ (m), 1525 (s), 1209 (s), 1016 (vs); ${ }^{1} \mathrm{H}$ NMR (250 MHz, CDCl $\left.{ }_{3}\right) \delta 9.31$ (d, J $9.3 \mathrm{~Hz}, 1 \mathrm{H}, H-\mathrm{Ar}$ ), 8.67 (d, J $2.3 \mathrm{~Hz}, 1 \mathrm{H}, H-\mathrm{Ar}$ ), 8.58 (d, J $9.8 \mathrm{~Hz}, 1 \mathrm{H}, H-2$ ), 8.45 (dd, J 9.3, $2.3 \mathrm{~Hz}, 1 \mathrm{H}, H-\mathrm{Ar}$ ), $4.45-4.15\left(\mathrm{~m}, 4 \mathrm{H}, \mathrm{CH}_{2} \mathrm{OP}\right), 1.40(\mathrm{t}, J 7.1 \mathrm{~Hz}, 6 \mathrm{H}) ;{ }^{13} \mathrm{C} \mathrm{NMR}\left(63 \mathrm{MHz}, \mathrm{CDCl}_{3}\right) \delta 166.4$ (s, C-Ar), 159.4 (d, J $11.0 \mathrm{~Hz}$, C-3), 158.6 (d, J $12.8 \mathrm{~Hz}, C(\mathrm{O})$ ), 146.1 (s, C-NO ) 139.5 (s, C-Ar), 125.6 (s, C-Ar), 122.8 (s, CH-Ar), 120.6 (s, CHAr), 117.9 (s, CH-Ar), 110.0 (d, J $197.3 \mathrm{~Hz}, \mathrm{C}-2$ ), 63.0 (d, J $5.9 \mathrm{~Hz}, \mathrm{CH}_{2} \mathrm{OP}$ ), 16.3 (d, J $\left.6.4 \mathrm{~Hz}, \mathrm{CH}_{3} \mathrm{CH}_{2} \mathrm{OP}\right) ;{ }^{31} \mathrm{P} \mathrm{NMR}$ $\left(101 \mathrm{MHz}, \mathrm{CDCl}_{3}\right) \delta$ 12.96. Anal. Calcd for $\mathrm{C}_{14} \mathrm{H}_{14} \mathrm{~N}_{3} \mathrm{O}_{6} \mathrm{PS}$ (383.31): C, 43.87; $\mathrm{H}, 3.68 ; \mathrm{N}$ 10.96\%. Found: $\mathrm{C}, 43.75$; $\mathrm{H}, 3.72$; N $10.92 \%$.

General procedure for the synthesis of 3-substituted $4 \mathrm{H}$-pyrimidobenzothiazol-4-ones $10 \mathrm{a}, \mathrm{d}, \mathrm{e}, \mathrm{i}-\mathrm{I}$. To a solution of the corresponding phosphonate $6 \mathrm{a}-\mathrm{c}(1 \mathrm{mmol})$ and a catalytic amount of Cul $(19 \mathrm{mg}, 0.1 \mathrm{mmol}) \mathrm{in}$ THF $(10 \mathrm{~mL})$ a solution of Grignard reagent $(5 \mathrm{mmol})$ was added dropwise, under an argon atmosphere at -78 ${ }^{\circ} \mathrm{C}$. The solution was stirred for $24 \mathrm{~h}$ at $\mathrm{rt}$. After this time the reaction mixture was quenched with $\mathrm{H}_{2} \mathrm{O}(2 \mathrm{~mL})$, acidified to $\mathrm{pH}$ ca. 1.5 with $10 \%$ aq $\mathrm{HCl}$ solution and extracted with $\mathrm{CHCl}_{3}(3 \times 10 \mathrm{~mL})$. The organic extracts were washed with brine $(10 \mathrm{~mL})$ and dried over $\mathrm{MgSO}_{4}$. Evaporation of the solvent gave the crude product which was purified by column chromatography (eluent: $\mathrm{CHCl}_{3}-\mathrm{MeOH}, 99: 1$ ).

Diethyl (2-methyl-4-oxo-3,4-dihydro-2H-benzo[4,5]thiazolo[3,2-a]pyrimidin-3-yl)phosphonate (10a). (80\%); yellow oil; IR v(cm $\left.{ }^{-1}\right)$ : 2926 (w), 1638 (s), 1467 (m), 1252 (s), 1012 (vs); $\left.{ }^{1} \mathrm{H} \mathrm{NMR} \mathrm{(250} \mathrm{MHz,} \mathrm{CDCl}_{3}\right) \delta 8.33-8.26$ (m, $1 \mathrm{H}$, major and minor, $H-A r), 7.34-7.16(\mathrm{~m}, 3 \mathrm{H}$, major and minor, $H-A r), 4.52$ (ddd, J 14.9, 6.9, 1.1 Hz, $1 \mathrm{H}$, major, $\mathrm{H}-2)$ ) $4.25-4.05\left(\mathrm{~m}, 5 \mathrm{H}\right.$, major and minor, $\mathrm{CH}_{2} \mathrm{OP}$, minor, $\left.\mathrm{H}-2\right), 3.30$ (dd, J 24.2, $\left.5.7 \mathrm{~Hz}, 1 \mathrm{H}, \mathrm{minor}, H-3\right)$, $3.08(\mathrm{dd}, J 24.8,1.1 \mathrm{~Hz}, 1 \mathrm{H}$, major, $\mathrm{H}-3), 1.34\left(\mathrm{t}, J 7.0 \mathrm{~Hz}, 3 \mathrm{H}\right.$, major and minor, $\left.\mathrm{CH}_{3} \mathrm{CH}_{2} \mathrm{O}\right), 1.31(\mathrm{t}, J 6.9 \mathrm{~Hz}, 3 \mathrm{H}$, major and minor, $\left.\mathrm{CH}_{3}\right), 1.24\left(\mathrm{t}, J 7.1 \mathrm{~Hz}, 3 \mathrm{H}\right.$, major and minor, $\left.\mathrm{CH}_{3} \mathrm{CH}_{2} \mathrm{O}\right) ;{ }^{13} \mathrm{C} \mathrm{NMR}\left(63 \mathrm{MHz}, \mathrm{CDCl}_{3}, \mathrm{major}\right) \delta$ 162.5 (d, J $5.1 \mathrm{~Hz}, \mathrm{C}(\mathrm{O})$ ), 153.8 (s, C-Ar), 135.2 (s, C-Ar), 126.1 (s, CH-Ar), 125.4 (s, CH-Ar), 122.4 (s, C-Ar), 121.3 (s, CH-Ar), 116.7 (s, CH-Ar), 63.1 (d, J 6.8 Hz, CH $2 \mathrm{OP}$ ), 62.8 (d, J $6.6 \mathrm{~Hz}, \mathrm{CH}_{2} \mathrm{OP}$ ), 52,0 (d, J 4.6 Hz, C-2), 46.5 (d, J $127.0 \mathrm{~Hz}, \mathrm{C}-3$ ), 20.5 (d, J $18.7 \mathrm{~Hz}, \mathrm{CH}_{3} \mathrm{CH}$ ), 15.9 (d, J $6.1 \mathrm{~Hz}, \mathrm{CH}_{3} \mathrm{CH}_{2} \mathrm{OP}$ ); ${ }^{31} \mathrm{P} \mathrm{NMR}\left(101 \mathrm{MHz}, \mathrm{CDCl}_{3}\right) \delta 19.93$ (major), 19.43 (minor). Anal. Calcd for $\mathrm{C}_{15} \mathrm{H}_{19} \mathrm{~N}_{2} \mathrm{O}_{4}$ PS (354.36): C, 50.84; H, 5.40; N 7.91\%. Found: $\mathrm{C}, 50.77 ; \mathrm{H}$, 5.42; N 7.95\%.

Diethyl (2-butyl-4-oxo-3,4-dihydro-2H-benzo[4,5]thiazolo[3,2-a]pyrimidin-3-yl)phosphonate (10d). (75\%); yellow oil; IR v(cm $\left.{ }^{-1}\right)$ : 2956 (w), 1646 (s), 1464 (m), 1248 (s), 1016 (vs); ${ }^{1} \mathrm{H} \mathrm{NMR} \mathrm{(250} \mathrm{MHz,} \mathrm{CDCl} 3$ ) $\delta 8.29-8.22$ (m, $1 \mathrm{H}$, major and minor, $H$-Ar), $7.34-7.13(\mathrm{~m}, 3 \mathrm{H}$, major and minor, $H$-Ar), $4.42-4.27$ (m, 2H, major and minor, $H-2$ ), $4.23-4.03\left(\mathrm{~m}, 4 \mathrm{H}\right.$, major and minor, $\mathrm{CH}_{2} \mathrm{OP}$ ), 3.27 (dd, J 24.0, $5.5 \mathrm{~Hz}, 1 \mathrm{H}$, minor, $H-3$ ), 3.10 (dd, J 25.1, $0.9 \mathrm{~Hz}, 1 \mathrm{H}$, major, $H-3), 1.78-1.58\left(\mathrm{~m}, 1 \mathrm{H}\right.$, major and minor, $\left.\mathrm{CH}_{2}\right), 1.56-1.26(\mathrm{~m}, 5 \mathrm{H}$, major and minor, 
$\mathrm{CH}_{2}$ ), $1.31\left(\mathrm{t}, J 7.1 \mathrm{~Hz}, 3 \mathrm{H}\right.$, major and minor, $\mathrm{CH}_{3} \mathrm{CH}_{2} \mathrm{O}$ ), $1.24\left(\mathrm{t}, J 7.1 \mathrm{~Hz}, 3 \mathrm{H}\right.$, major and minor, $\mathrm{CH}_{3} \mathrm{CH}_{2} \mathrm{O}$ ), 0.86 (t, J $7.1 \mathrm{~Hz}, 3 \mathrm{H}$, major and minor, $\left.\mathrm{CH}_{3} \mathrm{CH}_{2}\right) ;{ }^{13} \mathrm{C} \mathrm{NMR}\left(63 \mathrm{MHz}, \mathrm{CDCl}_{3}\right.$, major) $\delta 162.9$ (d, J 5.2 Hz, C(O)), $153.6(\mathrm{~s}$, C-Ar), 135.5 (s, C-Ar), 126.3 (s, CH-Ar), 125.7 (s, CH-Ar), 122.8 (s, C-Ar), 121.5 (s, CH-Ar), 117.0 (s, CH-Ar), 63.4 (d, J $6.8 \mathrm{~Hz}, \mathrm{CH}_{2} \mathrm{OP}$ ), 63.1 (d, J $6.5 \mathrm{~Hz}, \mathrm{CH}_{2} \mathrm{OP}$ ), 56.5 (d, J $4.7 \mathrm{~Hz}, \mathrm{C}-2$ ), 45.4 (d, J $126.9 \mathrm{~Hz}, \mathrm{C}-3$ ), 35.0 (d, J $17.2 \mathrm{~Hz}$, $\mathrm{CH}_{2} \mathrm{CH}$ ), 27.7 (s, $\mathrm{CH}_{2}$ ), $22.3\left(\mathrm{~s}, \mathrm{CH}_{2}\right.$ ), 16.2 (d, J $\left.\left.5.0 \mathrm{~Hz}, \mathrm{CH}_{3} \mathrm{CH}_{2} \mathrm{OP}\right), 13.8\left(\mathrm{~s}, \mathrm{CH}_{3}\right) ;{ }^{31} \mathrm{P} \mathrm{NMR}(101 \mathrm{MHz}, \mathrm{CDCl})\right) \delta$ 20.34 (major), 19.15 (minor). Anal. Calcd for $\mathrm{C}_{18} \mathrm{H}_{25} \mathrm{~N}_{2} \mathrm{O}_{4} \mathrm{PS}$ (396.44): C, 54.53; H, 6.36; N 7.07\%. Found: C, 54.45; H, 6.40; N 7.01\%.

Diethyl (4-oxo-2-phenyl-3,4-dihydro-2H-benzo[4,5]thiazolo[3,2-a]pyrimidin-3-yl)phosphonate (10e). (93\%); yellow oil; IR v(cm $\left.{ }^{-1}\right)$ : 2982 (w), 1643 (s), 1463 (m), 1279 (s), 1015 (vs); ${ }^{1} \mathrm{H} \mathrm{NMR} \mathrm{(250} \mathrm{MHz,} \mathrm{CDCl} 3$ ) $8.29-8.24$ (m, 1H, H-Ar), $7.34-7.13$ (m, 8H, H-Ar), 5.60 (dd, J 16.4, $1.2 \mathrm{~Hz}, 1 \mathrm{H}, \mathrm{H}-2), 4.28-4.13$ (m, 4H, CH $\mathrm{CPP}_{\text {) }} 3.46$ (dd, J 24.8, $1.2 \mathrm{~Hz}, 1 \mathrm{H}, \mathrm{H}-3$ ), 1.35 (t, J $\left.7.1 \mathrm{~Hz}, 3 \mathrm{H}, \mathrm{CH}_{3} \mathrm{CH}_{2} \mathrm{O}\right), 1.27\left(\mathrm{t}, J 7.1 \mathrm{~Hz}, 3 \mathrm{H}, \mathrm{CH}_{3} \mathrm{CH}_{2} \mathrm{O}\right) ;{ }^{13} \mathrm{C} \mathrm{NMR}(63 \mathrm{MHz}$, $\mathrm{CDCl}_{3}$ ) $\delta 162.2$ (d, J $5.3 \mathrm{~Hz}, \mathrm{C}(\mathrm{O})$ ), 155.8 (s, C-Ar), 139.4 (d, J 17.2 Hz, C-Ar), 135.5 (s, C-Ar), 129.0 (s, 2 x CH-Ar), 128.0 (s, CH-Ar), 126.4 (s, CH-Ar), 125.8 (s, CH-Ar), 125.8 (s, 2 x CH-Ar), 122.5 (s, C-Ar), 121.6 (s, CH-Ar), 117.1 (s, CH-Ar), 63.6 (d, J $\left.6.8 \mathrm{~Hz}, \mathrm{CH}_{2} \mathrm{OP}\right), 63.3$ (d, J $6.6 \mathrm{~Hz}, \mathrm{CH}_{2} \mathrm{OP}$ ), 59.6 (d, J $3.8 \mathrm{~Hz}, \mathrm{C}-2$ ), 47.6 (d, J 124.9 Hz, C-3), 16.2 (d, J $6.2 \mathrm{~Hz}, \mathrm{CH}_{3} \mathrm{CH}_{2} \mathrm{OP}$ ); ${ }^{31} \mathrm{P}$ NMR (101 MHz, $\left.\mathrm{CDCl}_{3}\right) \delta$ 19.64. Anal. Calcd for $\mathrm{C}_{20} \mathrm{H}_{21} \mathrm{~N}_{2} \mathrm{O}_{4} \mathrm{PS}$ (416.43): C, 57.68; H, 5.08; N 6.73\%. Found: C, 57.72; H, 5.14; N 6.75\%.

Diethyl (2-butyl-8-methyl-4-oxo-3,4-dihydro-2H-benzo[4,5]thiazolo[3,2-a]pyrimidin-3-yl)phosphonate (10i).

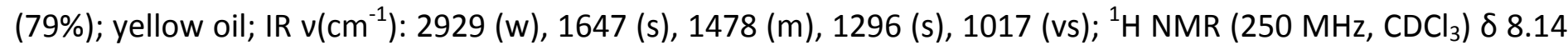
(d, J $8.4 \mathrm{~Hz}, 1 \mathrm{H}$, major and minor, $H-A r), 7.11(\mathrm{~d}, J 1.1 \mathrm{~Hz}, 1 \mathrm{H}$, major and minor, $H$-Ar), $7.04(\mathrm{dd}, J 8.4,1.1 \mathrm{~Hz}$, $1 \mathrm{H}$, major and minor, $H$-Ar), $4.44-4.26(\mathrm{~m}, 2 \mathrm{H}$, major and minor, $H-2), 4.24-4.04(\mathrm{~m}, 4 \mathrm{H}$, major and minor, $\mathrm{CH}_{2} \mathrm{OP}$ ), 3.27 (dd, J 24.1, $5.5 \mathrm{~Hz}, 1 \mathrm{H}$, minor, $\mathrm{H}-3$ ), 3.10 (dd, J 25.1, $1.0 \mathrm{~Hz}, 1 \mathrm{H}$, major, $\mathrm{H}-3$ ), $2.35\left(\mathrm{~s}, 3 \mathrm{H}, \mathrm{CH}_{3}\right), 1.79$ - $1.62\left(\mathrm{~m}, 2 \mathrm{H}\right.$, major and minor, $\left.\mathrm{CH}_{2}\right), 1.57-1.28\left(\mathrm{~m}, 4 \mathrm{H}\right.$, major and minor, $\left.\mathrm{CH}_{2}\right), 1.32(\mathrm{t}, J 7.1 \mathrm{~Hz}, 3 \mathrm{H}, \mathrm{major}$ and minor, $\left.\mathrm{CH}_{3} \mathrm{CH}_{2} \mathrm{O}\right), 1.23\left(\mathrm{t}, J 7.1 \mathrm{~Hz}, 3 \mathrm{H}\right.$, major and minor, $\left.\mathrm{CH}_{3} \mathrm{CH}_{2} \mathrm{O}\right), 0.88(\mathrm{t}, J 7.1 \mathrm{~Hz}, 3 \mathrm{H}$, major and minor, $\mathrm{CH}_{3} \mathrm{CH}_{2}$ ); ${ }^{13} \mathrm{CNMR}\left(63 \mathrm{MHz}, \mathrm{CDCl}_{3}\right.$, major) $\delta 162.7$ (d, J $5.2 \mathrm{~Hz}, \mathrm{C}(\mathrm{O})$ ), 153.9 (s, C-Ar), 135.7 (s, C-Ar), 133.3 (s, CAr), 126.9 (s, CH-Ar), 122.6 (s, C-Ar), 121.9 (s, CH-Ar), 116.7 (s, CH-Ar), 63.4 (d, J 6.7 Hz, CH $2 \mathrm{OP}$ ), 63.0 (d, J 6.7 $\mathrm{Hz}, \mathrm{CH}_{2} \mathrm{OP}$ ), 56.5 (d, J $4.7 \mathrm{~Hz}, \mathrm{C}-2$ ), 45.4 (d, J $126.9 \mathrm{~Hz}, \mathrm{C}-3$ ), 35.0 (d, J $17.2 \mathrm{~Hz}, \mathrm{CH}_{2} \mathrm{CH}$ ), 27.7 (s, CH$\left.)_{2}\right), 22.3(\mathrm{~s}$, $\mathrm{CH}_{2}$ ), 21.0 (s, $\mathrm{CH}_{3}$ ), 16.2 (d, J 4.2 Hz, $\mathrm{CH}_{3} \mathrm{CH}_{2} \mathrm{OP}$ ), 13.8 (s, $\mathrm{CH}_{3}$ ); ${ }^{31} \mathrm{P} \mathrm{NMR}$ (101 MHz, $\mathrm{CDCl}_{3}$ ) $\delta 20.50$ (major), 19.29 (minor). Anal. Calcd for $\mathrm{C}_{19} \mathrm{H}_{27} \mathrm{~N}_{2} \mathrm{O}_{4} \mathrm{PS}$ (410.47): C, 55.60; H, 6.63; N 6.82\%. Found: $\mathrm{C}, 55.39 ; \mathrm{H}, 6.68 ; \mathrm{N} 6.87 \%$.

Diethyl (8-methyl-4-oxo-2-phenyl-3,4-dihydro-2H-benzo[4,5]thiazolo[3,2-a]pyrimidin-3-yl)phosphonate (10j). (83\%); yellow oil; IR v(cm $\left.{ }^{-1}\right): 2982$ (w), 1643 (s), $1478(\mathrm{~m}), 1252$ (s), 1014 (vs); ${ }^{1} \mathrm{H} \mathrm{NMR} \mathrm{(250} \mathrm{MHz,} \mathrm{CDCl} \mathrm{MH}^{\circ}$ 8.13 (d, J $8.4 \mathrm{~Hz}, 1 \mathrm{H}, \mathrm{H}-\mathrm{Ar}$ ), $7.38-7.20$ (m, 5H, H-Ar), 7.16 (d, J $1.1 \mathrm{~Hz}, 1 \mathrm{H}, \mathrm{H}-\mathrm{Ar}$ ), 7.05 (dd, J 8.4, $1.1 \mathrm{~Hz}, 1 \mathrm{H}, \mathrm{H}-$ Ar), 5.58 (dd, J 16.4, $1.2 \mathrm{~Hz}, 1 \mathrm{H}, \mathrm{H}-2), 4.28-4.12\left(\mathrm{~m}, 4 \mathrm{H}, \mathrm{CH}_{2} \mathrm{OP}\right), 3.46$ (dd, J 24.8, $\left.1.2 \mathrm{~Hz}, 1 \mathrm{H}, H-3\right), 2.36(\mathrm{~s}, 1 \mathrm{H}$, $\left.\mathrm{CH}_{3}\right), 1.35\left(\mathrm{t}, J 7.1 \mathrm{~Hz}, 3 \mathrm{H}, \mathrm{CH}_{3} \mathrm{CH}_{2} \mathrm{O}\right), 1.27\left(\mathrm{t}, J 7.1 \mathrm{~Hz}, 3 \mathrm{H}, \mathrm{CH}_{3} \mathrm{CH}_{2} \mathrm{O}\right) ;{ }^{13} \mathrm{CNMR}\left(63 \mathrm{MHz}, \mathrm{CDCl}_{3}\right) \delta 161.9$ (d, J 5.1 $\mathrm{Hz}, \mathrm{C}(\mathrm{O})$ ), 156.0 (s, C-Ar), 139.5 (d, J $17.5 \mathrm{~Hz}, \mathrm{C}$-Ar), 135.9 (s, C-Ar), 133.2 (s, C-Ar), 128.9 (s, 2 x CH-Ar), 127.9 (s, CH-Ar), 127.0 (s, CH-Ar), 125.8 (s, 2 x CH-Ar), 122.4 (s, C-Ar), 121.9 (s, CH-Ar), 116.8 (s, CH-Ar), 63.5 (d, J $6.7 \mathrm{~Hz}$, $\mathrm{CH}_{2} \mathrm{OP}$ ), 63.3 (d, J $6.6 \mathrm{~Hz}, \mathrm{CH}_{2} \mathrm{OP}$ ), 59.6 (d, J $3.6 \mathrm{~Hz}, \mathrm{C}-2$ ), 47.6 (d, J $124.8 \mathrm{~Hz}, \mathrm{C}-3$ ), 21.0 (s, CH $\mathrm{CH}_{3}, 16.2$ (d, J $4.1 \mathrm{~Hz}$, $\mathrm{CH}_{3} \mathrm{CH}_{2} \mathrm{OP}$ ); ${ }^{31} \mathrm{P}$ NMR (101 MHz, CDCl $) \delta$ 19.77. Anal. Calcd for $\mathrm{C}_{21} \mathrm{H}_{23} \mathrm{~N}_{2} \mathrm{O}_{4} \mathrm{PS}$ (430.46): C, 58.59; $\mathrm{H}, 5.39 ; \mathrm{N}$ 6.51\%. Found: C, 58.52; $\mathrm{H}, 5.38 ; \mathrm{N} 6.47 \%$.

Diethyl (2-butyl-8-chloro-4-oxo-3,4-dihydro-2H-benzo[4,5]thiazolo[3,2-a]pyrimidin-3-yl)phosphonate (10k). (95\%); yellow oil; IR v(cm $\left.{ }^{-1}\right)$ : 2930 (w), 1645 (s), 1480 (m), 1292 (s), 1015 (vs); ${ }^{1} \mathrm{H}_{\mathrm{NMR}}\left(250 \mathrm{MHz}, \mathrm{CDCl}_{3}\right) \delta 8.20$ (d, J $8.8 \mathrm{~Hz}, 1 \mathrm{H}$, major and minor, $H$-Ar), 7.29 (d, J $1.1 \mathrm{~Hz}, 1 \mathrm{H}$, major and minor, $H$-Ar), 7.21 (dd, J 8.8, $1.1 \mathrm{~Hz}$, $1 \mathrm{H}$, major and minor, $H$-Ar), $4.45-4.29(\mathrm{~m}, 2 \mathrm{H}$, major and minor, $H-2), 4.24-4.04(\mathrm{~m}, 4 \mathrm{H}$, major and minor, $\mathrm{CH}_{2} \mathrm{OP}$ ), 3.27 (dd, J 24.0, $5.5 \mathrm{~Hz}, 1 \mathrm{H}$, minor, $H-3$ ), 3.11 (dd, J 25.1, $1.0 \mathrm{~Hz}, 1 \mathrm{H}$, major, $\left.H-3\right), 1.78-1.62(\mathrm{~m}, 2 \mathrm{H}$, 
major and minor, $\left.\mathrm{CH}_{2}\right), 1.56-1.28\left(\mathrm{~m}, 4 \mathrm{H}\right.$, major and minor, $\left.\mathrm{CH}_{2}\right), 1.33(\mathrm{t}, J 7.0 \mathrm{~Hz}, 3 \mathrm{H}$, major and minor, $\mathrm{CH}_{3} \mathrm{CH}_{2} \mathrm{O}$ ), $1.23\left(\mathrm{t}, \mathrm{J} 7.1 \mathrm{~Hz}, 3 \mathrm{H}\right.$, major and minor, $\left.\mathrm{CH}_{3} \mathrm{CH}_{2} \mathrm{O}\right), 0.88\left(\mathrm{t}, J 7.0 \mathrm{~Hz}, 3 \mathrm{H}\right.$, major and minor, $\left.\mathrm{CH}_{3} \mathrm{CH}_{2}\right) ;{ }^{13} \mathrm{C}$ NMR (63 MHz, CDCl , major) $\delta 162.8$ (d, J $5.3 \mathrm{~Hz}, C(O)$ ), 152.9 (s, C-Ar), 135.0 (s, C-Ar), 131.0 (s, C-Ar), 126.4 (s, $\mathrm{CH}-\mathrm{Ar}$ ), 124.6 (s, C-Ar), 121.4 (s, CH-Ar), 117.7 (s, CH-Ar), 63.4 (d, J $6.7 \mathrm{~Hz}, \mathrm{CH}_{2} \mathrm{OP}$ ), 63.1 (d, J 6.6 Hz, CH

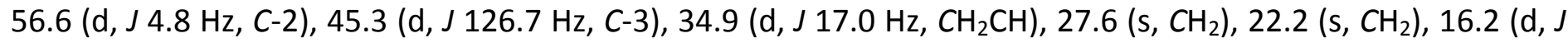
$6.2 \mathrm{~Hz}, \mathrm{CH}_{3} \mathrm{CH}_{2} \mathrm{OP}$ ), 13.7 (s, $\mathrm{CH}_{3}$ ); ${ }^{31} \mathrm{P} \mathrm{NMR}\left(101 \mathrm{MHz}, \mathrm{CDCl}_{3}\right) \delta 19.75$ (major), 18.49 (minor). Anal. Calcd for $\mathrm{C}_{18} \mathrm{H}_{24} \mathrm{ClN}_{2} \mathrm{O}_{4} \mathrm{PS}$ (430.88): C, 50.17; $\mathrm{H}, 5.61 ; \mathrm{N} 6.50 \%$. Found: C, 50.10; $\mathrm{H}, 5.64 ; \mathrm{N} 6.52 \%$.

Diethyl (8-chloro-4-oxo-2-phenyl-3,4-dihydro-2H-benzo[4,5]thiazolo[3,2-a]pyrimidin-3-yl)phosphonate (10I). (85\%); yellow oil; IR v(cm $\left.{ }^{-1}\right): 2980(\mathrm{w}), 1645$ (s), $1461(\mathrm{~m}), 1250(\mathrm{~m}), 1012$ (vs); ${ }^{1} \mathrm{H}$ NMR (250 MHz, CDCl $\left.{ }_{3}\right) 8.18$ (d, J $8.8 \mathrm{~Hz}, 1 \mathrm{H}, \mathrm{H}-\mathrm{Ar}$ ), $7.38-7.18$ (m, 7H, H-Ar), 5.60 (dd, J 16.4, 1.2 Hz, 1H, H-2), $4.27-4.11\left(\mathrm{~m}, 4 \mathrm{H}, \mathrm{CH} \mathrm{OP}^{\mathrm{O}}\right.$ ), 3.45 (dd, J 24.8, $1.2 \mathrm{~Hz}, 1 \mathrm{H}, \mathrm{H}-3$ ), 1.35 (t, J $\left.7.1 \mathrm{~Hz}, 3 \mathrm{H}, \mathrm{CH}_{3} \mathrm{CH}_{2} \mathrm{O}\right), 1.27\left(\mathrm{t}, J 7.1 \mathrm{~Hz}, 3 \mathrm{H}, \mathrm{CH}_{3} \mathrm{CH}_{2} \mathrm{O}\right) ;{ }^{13} \mathrm{C} \mathrm{NMR}(63$ $\mathrm{MHz} \mathrm{CDCl}_{3}$ ) $\delta 162.1$ (d, J $5.2 \mathrm{~Hz}, \mathrm{C}(\mathrm{O})$ ), 155.1 (s, C-Ar), 139.1 (d, J 17.3 Hz, C-Ar), 134.0 (s, C-Ar), 131.2 (s, C-Ar), 129.0 (s, 2 x CH-Ar), 128.1 (s, CH-Ar), 126.5 (s, CH-Ar), 125.7 (s, 2 x CH-Ar), 124.4 (s, C-Ar), 121.5 (s, CH-Ar), 117.8 (s, CH-Ar), 63.64(d, J $\left.6.9 \mathrm{~Hz}, \mathrm{CH}_{2} \mathrm{OP}\right), 63.4$ (d, J $6.6 \mathrm{~Hz}, \mathrm{CH}_{2} \mathrm{OP}$ ), 59.7 (d, J $4.0 \mathrm{~Hz}, \mathrm{C}-2$ ), 47.5 (d, J $124.7 \mathrm{~Hz}$, C-3), 16.2 (d, J $\left.6.1 \mathrm{~Hz}, \mathrm{CH}_{3} \mathrm{CH}_{2} \mathrm{OP}\right) ;{ }^{31} \mathrm{P} \mathrm{NMR}\left(101 \mathrm{MHz}, \mathrm{CDCl}_{3}\right) \delta$ 19.07. Anal. Calcd for $\mathrm{C}_{20} \mathrm{H}_{20} \mathrm{CIN}_{2} \mathrm{O}_{4} \mathrm{PS}(450.87)$ : C, 53.28; $\mathrm{H}, 4.47$; N 6.21\%. Found: C, 53.25; $H, 4.49 ; \mathrm{N} 6.21 \%$.

General procedure for the synthesis of 3-methylidene-4H-pyrimidobenzothiazol-4-ones $11 a, d, e, i-I$. To a solution of the corresponding $4 H$-pyrimidobenzothiazol-4-ones 10 a,d,e,i-I $(0.5 \mathrm{mmol})$ in THF (5 mL), NaH (14 $\mathrm{mg}, 0.6 \mathrm{mmol}$ ) was added and the resulting mixture was stirred at $\mathrm{rt}$ for $30 \mathrm{~min}$. Then, paraformaldehyde (75 $\mathrm{mg}, 2.5 \mathrm{mmol}$ ) was added in one portion. After $4 \mathrm{~h}$, the reaction mixture was quenched with brine $(10 \mathrm{~mL})$ and extracted with $\mathrm{CH}_{2} \mathrm{Cl}_{2}(3 \times 10 \mathrm{~mL})$. The organic layer was dried over $\mathrm{MgSO}_{4}$ and the solvent was evaporated. The crude product was purified by column chromatography (eluent: $\mathrm{CH}_{2} \mathrm{Cl}_{2}$ ).

2-Methyl-3-methylene-2,3-dihydro-4H-benzo[4,5]thiazolo[3,2-a]pyrimidin-4-one (11a). (51\%); yellow oil; IR $\mathrm{v}\left(\mathrm{cm}^{-1}\right): 2932$ (w), 1642 (vs), 1455 (s), 1247 (m), 745 (vs); ${ }^{1} \mathrm{H}$ NMR (250 MHz, CDCl 3 ) $88.40-8.27$ (m, 1H, H-Ar), $7.37-7.12$ (m, 3H, H-Ar), 6.45 (dd, J 1.9, $0.7 \mathrm{~Hz}, 1 \mathrm{H}, \mathrm{CH}_{2}=\mathrm{C}$ ), 5.69 (dd, J 2.0, $\left.0.7 \mathrm{~Hz}, 1 \mathrm{H}, \mathrm{CH}=\mathrm{C}\right), 4.61-4.43(\mathrm{~m}$, $1 \mathrm{H}, \mathrm{H}-2), 1.48\left(\mathrm{t}, J 6.9 \mathrm{~Hz}, 3 \mathrm{H}, \mathrm{CH}_{3}\right) ;{ }^{13} \mathrm{C} \mathrm{NMR}\left(63 \mathrm{MHz}, \mathrm{CDCl}_{3}\right) \delta 161.3(\mathrm{~s}, \mathrm{C}(\mathrm{O})), 153.4(\mathrm{~s}, \mathrm{C}-\mathrm{Ar}), 138.8(\mathrm{~s}, \mathrm{C}-\mathrm{Ar}$ ), 135.5 (s, C-Ar), 126.1 (s, CH-Ar), 125.6 (s, CH-Ar), 125.1 (s, CH l) $_{123.4}$ (s, C-Ar), 121.4 (s, CH-Ar), 117.7 (s, CHAr), 56.3 (s, C-2), 22.2 (s, $\mathrm{CH}_{3} \mathrm{CH}$ ). Anal. Calcd for $\mathrm{C}_{12} \mathrm{H}_{10} \mathrm{~N}_{2} \mathrm{OS}$ (230.29): C, 62.59; H, 4.38; N 12.16\%. Found: C, $62.51 ; \mathrm{H}, 4.42 ; \mathrm{N} 12.19 \%$.

2-Butyl-3-methylene-2,3-dihydro-4H-benzo[4,5]thiazolo[3,2-a]pyrimidin-4-one (11d). (34\%); yellow oil; IR $\mathrm{v}\left(\mathrm{cm}^{-1}\right): 2954$ (w), 1648 (vs), 1454 (s), 1246 (m), 746 (vs); ${ }^{1} \mathrm{H}$ NMR (250 MHz, CDCl $\left.{ }_{3}\right) \delta 8.37-8.28(\mathrm{~m}, 1 \mathrm{H}, \mathrm{H}-\mathrm{Ar})$, $7.35-7.13\left(\mathrm{~m}, 3 \mathrm{H}, \mathrm{H}-\mathrm{Ar}\right.$ ), 6.47 (dd, J 1.5, $1.0 \mathrm{~Hz}, 1 \mathrm{H}, \mathrm{CH}_{2}=\mathrm{C}$ ), 5.64 (dd, J 1.6, $\left.1.0 \mathrm{~Hz}, 1 \mathrm{H}, \mathrm{CH}_{2}=\mathrm{C}\right), 4.50-4.39$ (m, $1 \mathrm{H}, \mathrm{H}-2), 1.88-1.53\left(\mathrm{~m}, 2 \mathrm{H}, \mathrm{CH}_{2}\right), 1.50-1.22\left(\mathrm{~m}, 4 \mathrm{H}, 2 \times \mathrm{CH}_{2}\right), 0.90\left(\mathrm{t}, J 6.9 \mathrm{~Hz}, 3 \mathrm{H}, \mathrm{CH}_{3}\right) ;{ }^{13} \mathrm{C} \mathrm{NMR}(63 \mathrm{MHz}$, $\mathrm{CDCl}_{3}$ ) $\delta 161.5$ (s, C(O)), 153.3 (s, C-Ar), 137.8 (s, C-Ar), 135.7 (s, C-Ar), 126.3 (s, CH-Ar), 126.0 (s, CH-Ar), 125.7 (s, $\mathrm{CH}_{2}$ ), 123.6 (s, C-Ar), 121.5 (s, CH-Ar), 117.8 (s, CH-Ar), 61.5 (s, C-2), 36.2 (s, CH $\mathrm{CH}_{2}, 27.1$ (s, CH $\mathrm{CH}_{2}, 22.4\left(\mathrm{~s}, \mathrm{CH}_{2}\right.$ ), 13.9 (s, $\mathrm{CH}_{3}$ ). Anal. Calcd for $\mathrm{C}_{15} \mathrm{H}_{16} \mathrm{~N}_{2} \mathrm{OS}$ (272.37): C, 66.15; H, 5.92; N 10.29\%. Found: $\mathrm{C}, 66.02 ; \mathrm{H}, 5.93 ; \mathrm{N}$ $10.26 \%$.

3-Methylene-2-phenyl-2,3-dihydro-4H-benzo[4,5]thiazolo[3,2-a]pyrimidin-4-one (11e). (69\%); yellow oil; IR $\mathrm{v}\left(\mathrm{cm}^{-1}\right)$ : 2951 (w), 1640 (vs), 1451 (s), 1238 (m), 743 (vs); ${ }^{1} \mathrm{H}$ NMR (250 MHz, CDCl $) \delta 8.26-8.19$ (m, 1H, H-Ar), $7.58-7.01$ (m, 8H, H-Ar), $6.42\left(\mathrm{~d}, J 2.0 \mathrm{~Hz}, 1 \mathrm{H}, \mathrm{CH}_{2}=\mathrm{C}\right), 5.44(\mathrm{t}, J 2.0 \mathrm{~Hz}, 1 \mathrm{H}, \mathrm{H}-2), 5.40(\mathrm{~d}, J 2.0 \mathrm{~Hz}, 1 \mathrm{H}, \mathrm{CH}=\mathrm{C})$; ${ }^{13} \mathrm{C} \mathrm{NMR}\left(63 \mathrm{MHz}, \mathrm{CDCl}_{3}\right.$ ) $\delta 161.0$ (s, C(O)), 155.1 (s, C-Ar), 140.0 (s, C-Ar), 137.5 (s, C-Ar), 135.5 (s, C-Ar), 128.6

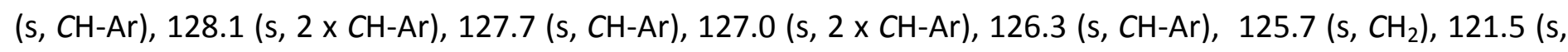


C-Ar), 119.6 (s, CH-Ar), 117.7 (s, CH-Ar), 64.4 (s, C-2). Anal. Calcd for $\mathrm{C}_{17} \mathrm{H}_{12} \mathrm{~N}_{2} \mathrm{OS}$ (292.36): C, 69.84; H, 4.14; N 9.58\%. Found: C, 69.80; $\mathrm{H}, 4.15 ; \mathrm{N} 9.61 \%$.

2-Butyl-8-methyl-3-methylene-2,3-dihydro-4H-benzo[4,5]thiazolo[3,2-a]pyrimidin-4-one (11i). (76\%); yellow oil; IR v(cm $\left.{ }^{-1}\right)$ : 2956 (w), 1645 (vs), 1454 (s), 1240 (m), 811 (vs); ${ }^{1} \mathrm{H}$ NMR (250 MHz, CDCl 3 ) $\delta 8.19$ (d, J $8.4 \mathrm{~Hz}$, 1H, H-Ar), 7.11 (d, J $1.1 \mathrm{~Hz}, 1 \mathrm{H}, H$-Ar), 7.04 (dd, J 8.4, $1.1 \mathrm{~Hz}, 1 \mathrm{H}, H-A r), 6.45$ (d, J $\left.1.2 \mathrm{~Hz}, 1 \mathrm{H}, \mathrm{CH}_{2}=\mathrm{C}\right), 5.62$ (d, J $\left.1.2 \mathrm{~Hz}, 1 \mathrm{H}, \mathrm{CH}_{2}=\mathrm{C}\right), 4.45-4.38(\mathrm{~m}, 1 \mathrm{H}, \mathrm{H}-2), 2.35\left(\mathrm{~s}, \mathrm{CH}_{3}\right), 1.83-1.55\left(\mathrm{~m}, 2 \mathrm{H}, \mathrm{CH}_{2}\right), 1.46-1.28\left(\mathrm{~m}, 4 \mathrm{H}, 2 \times \mathrm{CH}_{2}\right)$, $0.90\left(\mathrm{t}, \mathrm{J} 7.0 \mathrm{~Hz}, 3 \mathrm{H}, \mathrm{CH}_{3}\right) ;{ }^{13} \mathrm{C}$ NMR $\left(63 \mathrm{MHz}, \mathrm{CDCl}_{3}\right) \delta 161.4$ (s, C(O)), 153.5 (s, C-Ar), 137.8 (s, C-Ar), 135.7 (s,

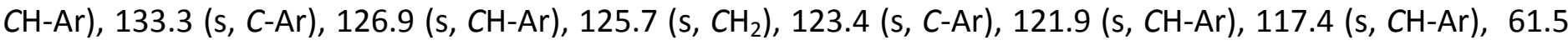
(s, C-2), $36.2\left(\mathrm{~s}, \mathrm{CH}_{2}\right), 27.1\left(\mathrm{~s}, \mathrm{CH}_{2}\right), 22.4\left(\mathrm{~s}, \mathrm{CH}_{2}\right), 21.1\left(\mathrm{~s}, \mathrm{CH}_{3}\right), 13.9$ (s, $\left.\mathrm{CH}_{3}\right)$. Anal. Calcd for $\mathrm{C}_{16} \mathrm{H}_{18} \mathrm{~N}_{2} \mathrm{OS}$ (286.39): C, 67.10; H, 6.34; N 9.78\%. Found: C, 67.01; H, 6.36; N 9.75\%.

8-Methyl-3-methylene-2-phenyl-2,3-dihydro-4H-benzo[4,5]thiazolo[3,2-a]pyrimidin-4-one (11j). (52\%);

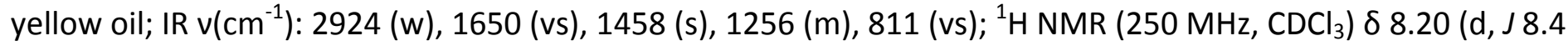
$\mathrm{Hz}, 1 \mathrm{H}, \mathrm{H}$-Ar), $7.40-7.28$ (m, 5H, H-Ar), $7.16-7.11$ (m, 1H, H-Ar), $7.11-7.03$ (m, 1H, H-Ar), 6.51 (dd, J 2.1, 0.8 $\mathrm{Hz}, 1 \mathrm{H}, \mathrm{CH}_{2}=\mathrm{C}$ ), 5.53 (t, J $\left.2.1 \mathrm{~Hz}, 1 \mathrm{H}, \mathrm{H}-2\right), 5.49$ (dd, J 2.1, $\left.0.8 \mathrm{~Hz}, 1 \mathrm{H}, \mathrm{CH}_{2}=\mathrm{C}\right), 2.36\left(\mathrm{~s}, \mathrm{CH}_{3}\right) ;{ }^{13} \mathrm{C} \mathrm{NMR}(63 \mathrm{MHz}$, $\mathrm{CDCl}_{3}$ ) $\delta 161.0$ (s, C(O)), 155.4 (s, C-Ar), 140.2 (s, C-Ar), 137.8 (s, C-Ar), 135.9 (s, CH-Ar), 133.3 (s, C-Ar), 128.7 (s, $2 \times \mathrm{CH}-\mathrm{Ar}$ ), 127.8 (s, CH-Ar), 127.8 (s, CH-Ar), 127.2 (s, $2 \times \mathrm{CH}-\mathrm{Ar}$ ), 127.0 (s, CH${ }_{2}$ ), 123.4 (s, C-Ar), 121.9 (s, C-Ar), 117.6 (s, CH-Ar), 64.6 (s, C-2), 21.1 (s, $\mathrm{CH}_{3}$ ). Anal. Calcd for $\mathrm{C}_{18} \mathrm{H}_{14} \mathrm{~N}_{2} \mathrm{OS}$ (306.38): C, 70.56; H, 4.61; N 9.14\%. Found: $\mathrm{C}, 70.48 ; \mathrm{H}, 4.64 ; \mathrm{N} 9.16 \%$.

2-Butyl-8-chloro-3-methylene-2,3-dihydro-4H-benzo[4,5]thiazolo[3,2-a]pyrimidin-4-one (11k). (78\%); yellow oil; IR v(cm ${ }^{-1}$ ): 2955 (w), 1648 (vs), 1506 (s), 1298 (m), 864 (vs); $\left.{ }^{1} \mathrm{H} \mathrm{NMR} \mathrm{(250} \mathrm{MHz,} \mathrm{CDCl}{ }_{3}\right) \delta 8.26$ (d, J $8.8 \mathrm{~Hz}$, $1 \mathrm{H}, H$-Ar), 7.28 (d, J $2.2 \mathrm{~Hz}, 1 \mathrm{H}, H$-Ar), 7.22 (dd, J 8.8, $2.2 \mathrm{~Hz}, 1 \mathrm{H}, H-\mathrm{Ar}$ ), 6.47 (d, J $\left.1.3 \mathrm{~Hz}, 1 \mathrm{H}, \mathrm{CH}_{2}=\mathrm{C}\right), 5.66(\mathrm{~d}, J$ $\left.1.4 \mathrm{~Hz}, 1 \mathrm{H}, \mathrm{CH}_{2}=\mathrm{C}\right), 4.49-4.38(\mathrm{~m}, 1 \mathrm{H}, \mathrm{H}-2), 1.83-1.56\left(\mathrm{~m}, 2 \mathrm{H}, \mathrm{CH}_{2}\right), 1.45-1.28\left(\mathrm{~m}, 4 \mathrm{H}, 2 \times \mathrm{CH}_{2}\right), 0.90(\mathrm{t}, J 7.0$ $\left.\mathrm{Hz}, 3 \mathrm{H}, \mathrm{CH}_{3}\right)$.

8-Chloro-3-methylene-2-phenyl-2,3-dihydro-4H-benzo[4,5]thiazolo[3,2-a]pyrimidin-4-one (11I). (84\%); yellow oil; 2917 (w), 1650 (vs), 1515 (s), 1181 (m), 751 (vs); ${ }^{1} \mathrm{H} \mathrm{NMR} \mathrm{(250} \mathrm{MHz,} \mathrm{CDCl} 3$ ) $\delta 8.19$ (d, J $8.8 \mathrm{~Hz}, 1 \mathrm{H}, \mathrm{H}-$ Ar), $7.37-7.19(\mathrm{~m}, 6 \mathrm{H}, H-\mathrm{Ar}), 7.19-7.14(\mathrm{~m}, 1 \mathrm{H}, H-\mathrm{Ar}), 6.46\left(\mathrm{~d}, J 2.0 \mathrm{~Hz}, 1 \mathrm{H}, \mathrm{CH}_{2}=\mathrm{C}\right), 5.47(\mathrm{t}, J 2.0 \mathrm{~Hz}, 1 \mathrm{H}, H-2)$, $5.45\left(\mathrm{~d}, \mathrm{~J} 2.0 \mathrm{~Hz}, 1 \mathrm{H}, \mathrm{CH}_{2}=\mathrm{C}\right)$.

General procedure for the preparation of diethyl (2-oxo-2H-benzothiazolopyrimidin-3-yl)phosphonates 9a,b,d. To a solution of ethyl 3-chloro-2-(diethoxyphosphoryl)acrylate 7 (10.0 mmol) in THF (50 mL) pyridine $(5$ $\mathrm{mL}$ ) and (2.66 g, $10.0 \mathrm{mmol}$ ) 2-aminobenzotiazole 3a, b, e were added and the mixture was stirred for 24 hours. After this time the reaction mixture was quenched with $\mathrm{H}_{2} \mathrm{O}(2 \mathrm{~mL})$, acidified to $\mathrm{pH}$ ca. 1.5 with $10 \%$ aq $\mathrm{HCl}$ solution and extracted with $\mathrm{CHCl}_{3}(3 \times 50 \mathrm{~mL})$. The organic extracts were washed with brine $(100 \mathrm{~mL})$ and dried over $\mathrm{MgSO}_{4}$. Evaporation of the solvent gave the crude product, which was purified by column chromatography (eluent: $\mathrm{CHCl}_{3}-\mathrm{MeOH}, 97: 3$ ).

Diethyl (2-oxo-2H-benzo[4,5]thiazolo[3,2-a]pyrimidin-3-yl)phosphonate (9a). (96\%); yellow crystals; mp 132$134{ }^{\circ} \mathrm{C}$; IR v(cm $\left.{ }^{-1}\right): 2987(\mathrm{w}), 1614(\mathrm{~s}), 1486(\mathrm{~m}), 1348(\mathrm{~m}), 1218(\mathrm{~m}), 981(\mathrm{vs}) ;{ }^{1} \mathrm{H} \mathrm{NMR}\left(250 \mathrm{MHz}, \mathrm{CDCl}_{3}\right) \delta 8.87$ (d, J $13.3 \mathrm{~Hz}, 1 \mathrm{H}, \mathrm{H}-4), 7.73-7.45(\mathrm{~m}, 4 \mathrm{H}, \mathrm{H}-\mathrm{Ar}), 4.42-4.17\left(\mathrm{~m}, 4 \mathrm{H}, \mathrm{CH}_{2} \mathrm{OP}\right), 1.38\left(\mathrm{t}, J 7.1 \mathrm{~Hz}, 6 \mathrm{H}, \mathrm{CH}_{3} \mathrm{CH}_{2} \mathrm{O}\right) ;{ }^{13} \mathrm{C}$ NMR (63 MHz, CDCl $) \delta 164.6$ (d, J 2.6 Hz, C(O)), 164.6 (s, C-Ar), 140.8 (d, J 17.3 Hz), 133.7 (s, C-Ar), 127.6 (s, CH-Ar), 127.1 (s, CH-Ar), 123.5 (s, C-Ar), 123.4 (s, CH-Ar), 111.8 (d, J 190.6 Hz, C-4), 111.7 (s, CH-Ar), 63.5 (d, J $6.1 \mathrm{~Hz}, \mathrm{CH}_{2} \mathrm{OP}$ ), 16.2 (d, J $6.4 \mathrm{~Hz}, \mathrm{CH}_{3} \mathrm{CH}_{2}$ ); ${ }^{31} \mathrm{P} \mathrm{NMR}\left(101 \mathrm{MHz}, \mathrm{CDCl}_{3}\right.$ ) $\delta$ 12.32. Anal. Calcd for $\mathrm{C}_{14} \mathrm{H}_{15} \mathrm{~N}_{2} \mathrm{O}_{4} \mathrm{PS}$ (338.32): C, 49.70; $\mathrm{H}, 4.47 ; \mathrm{N} 8.28 \%$. Found: $\mathrm{C}, 49.63 ; \mathrm{H}, 4.51 ; \mathrm{N} 8.25 \%$. Crystal data: formula $\mathrm{C}_{14} \mathrm{H}_{15} \mathrm{O}_{4} \mathrm{~N}_{2} \mathrm{P}_{1} \mathrm{~S}_{1}$ $\cdot 0.5 \mathrm{H}_{2} \mathrm{O}$, monoclinic, space group $P 2_{1} / c, Z=4$, cell constants $a=14.5768(5) \AA, b=14.1909(5) \AA, c=8.1019(3)$ $\AA, \beta=105.862(2)^{\circ}, V=1612.13(10) \AA^{3}$. The integration of the data yielded a total of 13768 reflections to a $\theta$ 
angle of $68.38^{\circ}$, of which 2928 were independent $\left(R_{\text {int }}=6.62 \%\right.$, ) and 2707 were greater than $2 \sigma\left(F^{2}\right)$. The final anisotropic full-matrix least-squares refinement on $F^{2}$ with 232 variables converged at $R_{1}=3.27 \%$, for the observed data and $\mathrm{wR}_{2}=9.30 \%$ for all data. All non-solvent hydrogen atoms, were placed in calculated positions and refined isotropically using a riding model. The disordered hydrogen atoms of water molecule placed around the inversion center were refined using suitable DFIX and DANG restrains. The goodness-of-fit was 1.044. CCDC 1477145 contains the supplementary crystallographic data for this paper. They can be obtained free of charge from The Cambridge Crystallographic Data Centre via www.ccdc.cam.ac.uk/data request/cif

Diethyl (8-methyl-2-oxo-2H-benzo[4,5]thiazolo[3,2-a]pyrimidin-3-yl)phosphonate (9b). (58\%); yellow crystals; mp 156-158 ${ }^{\circ} \mathrm{C}$; IR v(cm ${ }^{-1}$ ): 2983 (w), 1633 (vs), 1496 (s), 1275 (m), 1223 (s), 1051 (vs); ${ }^{1} \mathrm{H}$ NMR (250 $\mathrm{MHz}_{\mathrm{CDCl}}$ ) $\delta 8.87$ (d, J $\left.13.3 \mathrm{~Hz}, 1 \mathrm{H}, H-4\right), 7.53$ (d, J $8.3 \mathrm{~Hz}, 1 \mathrm{H}, H-\mathrm{Ar}$ ), 7.49 (d, J $1.1 \mathrm{~Hz}, 1 \mathrm{H}, H-\mathrm{Ar}$ ), 7.36 (dd, J 8.3, $1.1 \mathrm{~Hz}, 1 \mathrm{H}, \mathrm{H}-\mathrm{Ar}), 4.38-4.19\left(\mathrm{~m}, 4 \mathrm{H}, \mathrm{CH}_{2} \mathrm{OP}\right), 2.48\left(\mathrm{~s}, 3 \mathrm{H}, \mathrm{CH}_{3}\right), 1.37\left(\mathrm{t}, J 7.1 \mathrm{~Hz}, 6 \mathrm{H}, \mathrm{CH}_{3} \mathrm{CH}_{2} \mathrm{O}\right) ;{ }^{13} \mathrm{C} \mathrm{NMR}$ $\left(176 \mathrm{MHz}, \mathrm{CDCl}_{3}\right.$ ) $\delta 164.7$ (d, J $5.2 \mathrm{~Hz}, C(\mathrm{O})$ ), 164.6 (s, C-Ar), 140.9 (d, J $17.5 \mathrm{~Hz}$ ), 137.7 (s, C-Ar), 131.7 (s, C-Ar), 128.6 (s, CH-Ar), 123.6 (s, C-Ar), 123.4 (s, CH-Ar), 111.7 (d, J 190.5 Hz, C-4), 111.4 (s, CH-Ar), 63.6 (d, J 6.0 Hz, $\mathrm{CH}_{2} \mathrm{OP}$ ), 16.3 (d, J $6.4 \mathrm{~Hz}, \mathrm{CH}_{3} \mathrm{CH}_{2}$ ); ${ }^{31} \mathrm{P} \mathrm{NMR}\left(101 \mathrm{MHz}, \mathrm{CDCl}_{3}\right) \delta$ 12.39. Anal. Calcd for $\mathrm{C}_{15} \mathrm{H}_{17} \mathrm{~N}_{2} \mathrm{O}_{4} \mathrm{PS}$ (352.34): C, 51.13; H, 4.86; N 7.95\%. Found: C, 51.07; H, 4.90; N 8.01\%.

Diethyl (8-methoxy-2-oxo-2H-benzo[4,5]thiazolo[3,2-a]pyrimidin-3-yl)phosphonate (9e). (76\%); yellow crystals; mp 176-178 ${ }^{\circ} \mathrm{C}$; IR v(cm ${ }^{-1}$ ): 2983 (w), 1628 (vs), 1496 (vs), 1338 (s), 1237 (m), 1020 (vs); ${ }^{1} \mathrm{H}$ NMR (700 $\mathrm{MHz} \mathrm{CDCl}_{3}$ ) $\delta 8.78$ (d, J $\left.13.2 \mathrm{~Hz}, 1 \mathrm{H}, H-4\right), 7.54$ (d, J $9.1 \mathrm{~Hz}, 1 \mathrm{H}, H-\mathrm{Ar}$ ), 7.16 (d, J $\left.2.5 \mathrm{~Hz}, 1 \mathrm{H}, H-\mathrm{Ar}\right), 7.07$ (dd, J 9.1, $2.5 \mathrm{~Hz}, 1 \mathrm{H}, \mathrm{H}-\mathrm{Ar}), 4.33-4.27\left(\mathrm{~m}, 2 \mathrm{H}, \mathrm{CH}_{2} \mathrm{OP}\right), 4.26-4.20\left(\mathrm{~m}, 2 \mathrm{H}, \mathrm{CH}_{2} \mathrm{OP}\right), 3.87\left(\mathrm{~s}, 3 \mathrm{H}, \mathrm{CH}_{3}\right), 1.35(\mathrm{t}, J 7.1$ $\left.\mathrm{Hz}, 6 \mathrm{H}, \mathrm{CH}_{3} \mathrm{CH}_{2} \mathrm{O}\right) ;{ }^{13} \mathrm{C}$ NMR (176 MHz, CDCl 3 ) $\delta 164.5$ (d, J 5.1 Hz, C(O)), 164.3 (s, C-Ar), 158.8 (s, C-OMe), 140.9 (d, J 17.7 Hz), 127.6 (s, C-Ar), 125.1 (s, C-Ar), 114.7 (s, CH-Ar), 112.6 (s, CH-Ar), 111.6 (d, J 190.1 Hz, C-4), 107.7 (s, CH-Ar), 63.6 (d, J $6.1 \mathrm{~Hz}, \mathrm{CH}_{2} \mathrm{OP}$ ), 56.0 (s, $\mathrm{CH}_{3} \mathrm{O}$ ), 16.3 (d, J $6.4 \mathrm{~Hz}, \mathrm{CH}_{3} \mathrm{CH}_{2}$ ); ${ }^{31} \mathrm{P} \mathrm{NMR}(283 \mathrm{MHz}, \mathrm{CDCl}$ ) $\delta$ 12.61. Anal. Calcd for $\mathrm{C}_{15} \mathrm{H}_{17} \mathrm{~N}_{2} \mathrm{O}_{5} \mathrm{PS}$ (368.34): C, 48.91; $\mathrm{H}, 4.65 ; \mathrm{N} 7.61 \%$. Found: $\mathrm{C}, 48.81 ; \mathrm{H}, 4.64 ; \mathrm{N} 7.57 \%$.

General Procedure for the Synthesis of 4-substituted diethyl (2-oxo-3,4-dihydro-2H-benzothiazolopyrimidin3-yl)phosphonates $\mathbf{1 2 a - j , m}$. To a solution of the corresponding phosphonate $\mathbf{9 a}, \mathbf{b}, \mathbf{e}(1 \mathrm{mmol})$ in THF (10 mL) a solution of Grignard reagent ( $5 \mathrm{mmol}$ ) was added dropwise, under an argon atmosphere at $0{ }^{\circ} \mathrm{C}$. The solution was stirred for $24 \mathrm{~h}$ at $\mathrm{rt}$. After this time the reaction mixture was quenched with $\mathrm{H}_{2} \mathrm{O}(2 \mathrm{~mL})$, acidified to $\mathrm{pH}$ ca. 1.5 with $10 \%$ aq $\mathrm{HCl}$ solution and extracted with $\mathrm{CHCl}_{3}(3 \times 10 \mathrm{~mL})$. The organic extracts were washed with brine $(10 \mathrm{~mL})$ and dried over $\mathrm{MgSO}_{4}$. Evaporation of the solvent gave the crude product, which was purified by column chromatography (eluent: $\left.\mathrm{CHCl}_{3}-\mathrm{MeOH}, 99: 1\right)$.

Diethyl (4-methyl-2-oxo-3,4-dihydro-2H-benzo[4,5]thiazolo[3,2-a]pyrimidin-3-yl)phosphonate (12a). (73\%); yellow crystals; mp 144-146 ${ }^{\circ} \mathrm{C}$; IR v(cm $\left.{ }^{-1}\right)$ : $2983(\mathrm{w}), 1672$ (s), 1505 (vs), 1345 (m), 1236 (m), 1012 (s); ${ }^{1} \mathrm{H}$ NMR $\left(250 \mathrm{MHz}, \mathrm{CDCl}_{3}\right) \delta 7.57$ (d, J $7.7 \mathrm{~Hz}, 1 \mathrm{H}, H$-Ar), $7.50-7.39(\mathrm{~m}, 1 \mathrm{H}, H-\mathrm{Ar}), 7.32-7.19(\mathrm{~m}, 2 \mathrm{H}, H-\mathrm{Ar}), 5.04$ (dqd, J 13.6, 6.8, $0.8 \mathrm{~Hz}, 1 \mathrm{H}, \mathrm{H}-4), 4.23-4.08\left(\mathrm{~m}, 2 \mathrm{H}, \mathrm{CH}_{2} \mathrm{OP}\right), 3.98-3.83\left(\mathrm{~m}, 2 \mathrm{H}, \mathrm{CH}_{2} \mathrm{OP}\right), 3.15(\mathrm{dd}, J 23.6,0.8 \mathrm{~Hz}, 1 \mathrm{H}$, $\mathrm{H}-3$ ), $1.47\left(\mathrm{~d}, J 6.8 \mathrm{~Hz}, 3 \mathrm{H}, \mathrm{CH}_{3} \mathrm{CH}\right), 1.31\left(\mathrm{t}, J 7.1 \mathrm{~Hz}, 3 \mathrm{H}, \mathrm{CH}_{3} \mathrm{CH}_{2} \mathrm{O}\right), 0.96\left(\mathrm{t}, J 7.1 \mathrm{~Hz}, 3 \mathrm{H}, \mathrm{CH}_{3} \mathrm{CH}_{2} \mathrm{O}\right) ;{ }^{13} \mathrm{C} \mathrm{NMR}(176$ $\mathrm{MHz} \mathrm{CDCl}_{3}$ ) $\delta 171.7$ (s, C-Ar), 168.1 (d, J $4.5 \mathrm{~Hz}, \mathrm{C}(\mathrm{O})$ ), 137.3 (s, C-Ar), 127.4 (s, CH-Ar), 124.3 (s, CH-Ar), 123.5 (s, C-Ar), 122.8 (s, CH-Ar), 110.6 (s, CH-Ar), 63.5 (d, J $6.1 \mathrm{~Hz}, \mathrm{CH}_{2} \mathrm{OP}$ ), 62.9 (d, J $6.1 \mathrm{~Hz}, \mathrm{CH}_{2} \mathrm{OP}$ ), 49.5 (d, J $5.9 \mathrm{~Hz}$, C-4), 45.4 (d, J $126.1 \mathrm{~Hz}, C-3), 18.9$ (d, J17.7 Hz, CH $), 16.1$ (d, J $6.3 \mathrm{~Hz}, C_{3} \mathrm{CH}_{2}$ ), $15.8\left(\mathrm{~d}, J 6.4 \mathrm{~Hz}_{3} \mathrm{CH}_{3} \mathrm{CH}_{2}\right.$ ); ${ }^{31} \mathrm{P}$ NMR (283 MHz, $\mathrm{CDCl}_{3}$ ) $\delta$ 19.37. Anal. Calcd for $\mathrm{C}_{15} \mathrm{H}_{19} \mathrm{~N}_{2} \mathrm{O}_{4} \mathrm{PS}$ (354.36): C, 50.84; $\mathrm{H}, 5.40 ; \mathrm{N}$ 7.91\%. Found: C, 50.84; H, 5.43; N 7.96\%.

Diethyl (4-ethyl-2-oxo-3,4-dihydro-2H-benzo[4,5]thiazolo[3,2-a]pyrimidin-3-yl)phosphonate (12b). (68\%); yellow crystals; mp $128-130{ }^{\circ} \mathrm{C} ; \mathrm{IR} \mathrm{v}\left(\mathrm{cm}^{-1}\right)$ : $2961(\mathrm{w}), 1737(\mathrm{~m}), 1497(\mathrm{vs}), 1348(\mathrm{~m}), 1230(\mathrm{~m}), 1013(\mathrm{~s}) ;{ }^{1} \mathrm{H}$ NMR 
$\left(700 \mathrm{MHz}, \mathrm{CDCl}_{3}\right) \delta 7.55$ (d, J $\left.7.8 \mathrm{~Hz}, 1 \mathrm{H}, H-\mathrm{Ar}\right), 7.42$ (d, J $7.8 \mathrm{~Hz}, 1 \mathrm{H}, H-\mathrm{Ar}$ ), 7.24 (d, J $\left.7.8 \mathrm{~Hz}, 1 \mathrm{H}, H-\mathrm{Ar}\right), 7.20$ (d, J $7.8 \mathrm{~Hz}, 1 \mathrm{H}, \mathrm{H}-\mathrm{Ar}$ ), $4.82(\mathrm{dt}, J \mathrm{l}$ 14.0, $6.6 \mathrm{~Hz}, 1 \mathrm{H}, \mathrm{H}-4), 4.19-4.12\left(\mathrm{~m}, 2 \mathrm{H}, \mathrm{CH}_{2} \mathrm{OP}\right), 3.93-3.86(\mathrm{~m}, 2 \mathrm{H}, \mathrm{CH} \mathrm{OP}), 3.24$ (d, J $24.0 \mathrm{~Hz}, 1 \mathrm{H}, \mathrm{H}-3$ ), $1.91-1.79\left(\mathrm{~m}, 2 \mathrm{H}, \mathrm{CH}_{3} \mathrm{CH}_{2}\right), 1.30\left(\mathrm{~d}, J 7.0 \mathrm{~Hz}, 3 \mathrm{H}, \mathrm{CH}_{3} \mathrm{CH}_{2} \mathrm{O}\right), 0.98(\mathrm{t}, J 7.4 \mathrm{~Hz}, 3 \mathrm{H}$, $\mathrm{CH}_{3} \mathrm{CH}$ ), 0.94 (t, J $7.0 \mathrm{~Hz}, 3 \mathrm{H}, \mathrm{CH}_{3} \mathrm{CH}_{2} \mathrm{O}$ ); ${ }^{13} \mathrm{C} \mathrm{NMR}\left(176 \mathrm{MHz}, \mathrm{CDCl}_{3}\right.$ ) $\delta 172.8$ (s, C-Ar), 168.4 (d, J $4.5 \mathrm{~Hz}, \mathrm{C}(\mathrm{O})$ ), 137.9 (s, C-Ar), 127.4 (s, CH-Ar), 124.2 (s, CH-Ar), 123.5 (s, C-Ar), 122.7 (s, CH-Ar), 111.1 (s, CH-Ar), 63.6 (d, J 6.6 $\mathrm{Hz}, \mathrm{CH}_{2} \mathrm{OP}$ ), 62.9 (d, J $6.6 \mathrm{~Hz}, \mathrm{CH}_{2} \mathrm{OP}$ ), 54.8 (d, J $5.9 \mathrm{~Hz}, \mathrm{C}-4$ ), 42.8 (d, J $127.0 \mathrm{~Hz}, \mathrm{C}-3$ ), 26.4 (d, J 16.4 Hz, $\mathrm{CH}_{3} \mathrm{CH}_{2}$ ), 16.2 (d, J $6.3 \mathrm{~Hz}, \mathrm{CH}_{3} \mathrm{CH}_{2}$ ), 15.9 (d, J $6.5 \mathrm{~Hz}, \mathrm{CH}_{3} \mathrm{CH}_{2}$ ), $9.6\left(\mathrm{~s}, \mathrm{CH}_{3}\right) ;{ }^{31} \mathrm{P} \mathrm{NMR}\left(283 \mathrm{MHz}, \mathrm{CDCl}_{3}\right) \delta$ 19.89. Anal. Calcd for $\mathrm{C}_{16} \mathrm{H}_{21} \mathrm{~N}_{2} \mathrm{O}_{4} \mathrm{PS}$ (368.39): C, 52.17; H, 5.75; N 7.60\%. Found: $\mathrm{C}, 52.17 ; \mathrm{H}, 5.77 ; \mathrm{N} 7.64 \%$.

Diethyl (4-isopropyl-2-oxo-3,4-dihydro-2H-benzo[4,5]thiazolo[3,2-a]pyrimidin-3-yl)phosphonate (12c). (43\%); yellow crystals; mp 152-154 ${ }^{\circ} \mathrm{C}$; IR v(cm $\left.{ }^{-1}\right): 2967$ (w), 1658 (m), 1493 (vs), 1367 (s), 1240 (m), 1016 (s); ${ }^{1} \mathrm{H}$ NMR (250 MHz, CDCl $) \delta 7.58(\mathrm{~d}, J 7.9 \mathrm{~Hz}, 1 \mathrm{H}, \mathrm{H}-\mathrm{Ar}), 7.45$ (d, J 7.9 Hz, 1H, H-Ar), 7.29 (d, J 7.9 Hz, 1H, H-Ar), 7.22 (d, J $7.9 \mathrm{~Hz}, 1 \mathrm{H}, \mathrm{H}-\mathrm{Ar}), 4.73$ (dd, J 17.3, $5.3 \mathrm{~Hz}, 1 \mathrm{H}, \mathrm{H}-4), 4.28-4.09$ (m, 2H, CH. OP), $4.04-3.82(\mathrm{~m}, 2 \mathrm{H}$, $\mathrm{CH}_{2} \mathrm{OP}$ ), 3.32 (d, J $\left.24.9 \mathrm{~Hz}, 1 \mathrm{H}, \mathrm{H}-3\right), 2.46-2.26\left(\mathrm{~m}, 1 \mathrm{H},\left(\mathrm{CH}_{3}\right)_{2} \mathrm{CH}\right), 1.33\left(\mathrm{~d}, J 7.1 \mathrm{~Hz}, 3 \mathrm{H}, \mathrm{CH}_{3} \mathrm{CH}_{2} \mathrm{O}\right), 1.03(\mathrm{~d}, J 7.2$ $\left.\mathrm{Hz}, 3 \mathrm{H}, \mathrm{CH}_{3} \mathrm{CH}\right), 0.98\left(\mathrm{~d}, J 6.8 \mathrm{~Hz}, 3 \mathrm{H}, \mathrm{CH}_{3} \mathrm{CH}\right), 0.95\left(\mathrm{t}, J 7.1 \mathrm{~Hz}, 3 \mathrm{H}, \mathrm{CH}_{3} \mathrm{CH}_{2} \mathrm{O}\right) ;{ }^{13} \mathrm{C} \mathrm{NMR}(176 \mathrm{MHz}, \mathrm{CDCl} 3) \delta$ 172.9 (s, C-Ar), 168.4 (d, J 5.0 Hz, C(O)), 138.2 (s, C-Ar), 127.2 (s, CH-Ar), 124.1 (s, CH-Ar), 123.3 (s, C-Ar), 122.6 (s, CH-Ar), 111.1 (s, CH-Ar), 63.6 (d, J $6.6 \mathrm{~Hz}, \mathrm{CH}_{2} \mathrm{OP}$ ), 62.9 (d, J $6.6 \mathrm{~Hz}, \mathrm{CH}_{2} \mathrm{OP}$ ), 54.8 (d, J $5.9 \mathrm{~Hz}, \mathrm{C}-4$ ), 42.8 (d, J $127.0 \mathrm{~Hz}, \mathrm{C}-3$ ), 26.4 (d, J16.4 Hz, CH${ }_{3} \mathrm{CH}$ ), 16.2 (d, J $6.3 \mathrm{~Hz}, \mathrm{CH}_{3} \mathrm{CH}_{2}$ ), 15.9 (d, J $\left.6.5 \mathrm{~Hz}, \mathrm{CH}_{3} \mathrm{CH}_{2}\right), 9.6\left(\mathrm{~s}, \mathrm{CH}_{3}\right) ;{ }^{31} \mathrm{P}$ NMR (283 MHz, $\mathrm{CDCl}_{3}$ ) $\delta$ 19.89. Anal. Calcd for $\mathrm{C}_{17} \mathrm{H}_{23} \mathrm{~N}_{2} \mathrm{O}_{4} \mathrm{PS}$ (382.41): C, 53.39; $\mathrm{H}, 6.06 ; \mathrm{N} 7.33 \%$. Found: C, 53.30; $\mathrm{H}, 6.10 ; \mathrm{N} 7.35 \%$.

Diethyl (4-butyl-2-oxo-3,4-dihydro-2H-benzo[4,5]thiazolo[3,2-a]pyrimidin-3-yl)phosphonate (12d). (72\%); yellow crystals; mp 122-124 ${ }^{\circ} \mathrm{C}$; IR v(cm $\left.{ }^{-1}\right)$ : $2959(\mathrm{w}), 1670(\mathrm{~m}), 1497(\mathrm{vs}), 1348(\mathrm{~m}), 1244(\mathrm{~m}), 1016(\mathrm{~s}) ;{ }^{1} \mathrm{H}$ NMR $\left(700 \mathrm{MHz} \mathrm{CDCl}_{3}\right) \delta 7.58$ (d, J $7.9 \mathrm{~Hz}, 1 \mathrm{H}, \mathrm{H}-\mathrm{Ar}$ ), $7.49-7.44(\mathrm{~m}, 1 \mathrm{H}, \mathrm{H}-\mathrm{Ar}), 7.31-7.26(\mathrm{~m}, 1 \mathrm{H}, H-\mathrm{Ar}), 7.23(\mathrm{~d}, J$ $8.2 \mathrm{~Hz}, 1 \mathrm{H}, \mathrm{H}-\mathrm{Ar}), 4.73$ (dtd, J 14.6, 6.9, $0.5 \mathrm{~Hz}, 1 \mathrm{H}, \mathrm{H}-4), 4.22-4.16(\mathrm{~m}, 2 \mathrm{H}, \mathrm{CH} \mathrm{OP}), 3.95-3.90(\mathrm{~m}, 2 \mathrm{H}$, $\mathrm{CH}_{2} \mathrm{OP}$ ), 3.28 (dd, J 24.1, $\left.0.5 \mathrm{~Hz}, 1 \mathrm{H}, \mathrm{H}-3\right), 1.83-1.81\left(\mathrm{~m}, 2 \mathrm{H}, \mathrm{CH}_{2}\right), 1.44-1.35\left(\mathrm{~m}, 4 \mathrm{H}, 2 \times \mathrm{CH}_{2}\right), 1.34(\mathrm{~d}, J 7.0$ $\left.\mathrm{Hz}, 3 \mathrm{H}, \mathrm{CH}_{3} \mathrm{CH}_{2} \mathrm{O}\right), 0.97\left(\mathrm{~d}, J 7.0 \mathrm{~Hz}, 3 \mathrm{H}, \mathrm{CH}_{3} \mathrm{CH}_{2} \mathrm{O}\right), 0.88\left(\mathrm{~d}, J 7.1 \mathrm{~Hz}, 3 \mathrm{H}, \mathrm{CH}_{3} \mathrm{CH}\right) ;{ }^{13} \mathrm{C} \mathrm{NMR}\left(176 \mathrm{MHz}^{\mathrm{CDCl}}\right)_{3} \delta$ 171.9 (s, C-Ar), 168.1 (d, J 4.4 Hz, C(O)), 137.4 (s, C-Ar), 127.1 (s, CH-Ar), 123.9 (s, CH-Ar), 123.1 (s, C-Ar), 122.5 (s, CH-Ar), 110.7 (s, CH-Ar), 63.1 (d, J 6.5 Hz, CH $2 \mathrm{OP}$ ), 62.6 (d, J 7.2 Hz, CH ${ }_{2} \mathrm{OP}$ ), 53.3 (d, J $6.7 \mathrm{~Hz}, \mathrm{C}-4$ ), 42.7 (d, J $126.8 \mathrm{~Hz}, \mathrm{C}-3$ ), 32.3 (d, J $16.0 \mathrm{~Hz}, \mathrm{CH}_{2} \mathrm{CH}$ ), 26.6 (s, $\mathrm{CH}_{2} \mathrm{CH}_{2}$ ), 21.9 (s, $\mathrm{CH}_{2} \mathrm{CH}_{2}$ ), 15.8 (d, J $\left.5.8 \mathrm{~Hz}, \mathrm{CH}_{3} \mathrm{CH}_{2} \mathrm{O}\right), 15.9$ (d, J $\left.6.0 \mathrm{~Hz}, \mathrm{CH}_{3} \mathrm{CH}_{2} \mathrm{O}\right), 13.3\left(\mathrm{~s}, \mathrm{CH}_{3} \mathrm{CH}_{2}\right) ;{ }^{31} \mathrm{P} \mathrm{NMR}\left(283 \mathrm{MHz}, \mathrm{CDCl}_{3}\right) \delta$ 19.53. Anal. Calcd for $\mathrm{C}_{18} \mathrm{H}_{25} \mathrm{~N}_{2} \mathrm{O}_{4} \mathrm{PS}$ (396.44): C, 54.53; H, 6.36; N 7.07\%. Found: C, 54.42; H, 6.37; N 7.04\%.

Diethyl (2-oxo-4-phenyl-3,4-dihydro-2H-benzo[4,5]thiazolo[3,2-a]pyrimidin-3-yl)phosphonate (12e). (68\%); yellow crystals; mp 175-177 ${ }^{\circ} \mathrm{C}$; IR v(cm $\left.{ }^{-1}\right)$ : 2980 (w), 1671 (m), 1494 (vs), 1354 (s), 1236 (s), 1023 (vs); ${ }^{1} \mathrm{H} \mathrm{NMR}$ $\left(700 \mathrm{MHz} \mathrm{CDCl}_{3}\right) \delta 7.62-7.56(\mathrm{~m}, 1 \mathrm{H}, \mathrm{H}-\mathrm{Ar}), 7.38-7.29$ (m, 4H, H-Ar), $7.28-7.18$ (m, 3H, H-Ar), $7.10-7.04$ (m, 1H, H-Ar), 5.95 (dd, J 17.2, $0.8 \mathrm{~Hz}, 1 \mathrm{H}, \mathrm{H}-4), 4.29-4.15\left(\mathrm{~m}, 2 \mathrm{H}, \mathrm{CH}_{2} \mathrm{OP}\right), 4.09-3.95\left(\mathrm{~m}, 2 \mathrm{H}, \mathrm{CH} \mathrm{OP}_{2}\right), 3.40$ (dd, J 23.4, $0.8 \mathrm{~Hz}, 1 \mathrm{H}, \mathrm{H}-3), 1.36$ (d, J $\left.7.1 \mathrm{~Hz}, 3 \mathrm{H}, \mathrm{CH}_{3} \mathrm{CH}_{2} \mathrm{O}\right), 1.06$ (d, J $\left.7.1 \mathrm{~Hz}, 3 \mathrm{H}, \mathrm{CH}_{3} \mathrm{CH}_{2} \mathrm{O}\right) ;{ }^{13} \mathrm{C} \mathrm{NMR}(176$ $\mathrm{MHz} \mathrm{CDCl}_{3}$ ) $\delta 173.0$ (s, C-Ar), 167.4 (d, J $5.0 \mathrm{~Hz}, \mathrm{C}(\mathrm{O})$ ), 137.6 (s, C-Ar), 136.6 (d, J $15.9 \mathrm{~Hz}, \mathrm{C}-\mathrm{Ar}$ ), 129.6 (s, 2 x CH-Ar), 129.1 (s, CH-Ar), 127.4 (s, CH-Ar), 125.2 (s, 2 x CH-Ar), 124.5 (s, CH-Ar), 123.1 (s, C-Ar), 122.6 (s, CH-Ar), 111.4 (s, CH-Ar), 63.7 (d, J 6.5 Hz, CH ${ }_{2} \mathrm{OP}$ ), 63.1 (d, J $7.1 \mathrm{~Hz}, \mathrm{CH}_{2} \mathrm{OP}$ ), 56.7 (d, J $5.3 \mathrm{~Hz}, \mathrm{C}-4$ ), 47.0 (d, J $123.4 \mathrm{~Hz}$, C-3), 16.1 (d, J $6.1 \mathrm{~Hz}, \mathrm{CH}_{3} \mathrm{CH}_{2} \mathrm{O}$ ), 15.9 (d, J $6.3 \mathrm{~Hz}, \mathrm{CH}_{3} \mathrm{CH}_{2} \mathrm{O}$ ); ${ }^{31} \mathrm{P} \mathrm{NMR}\left(283 \mathrm{MHz}, \mathrm{CDCl}_{3}\right) \delta$ 19.13. Anal. Calcd for $\mathrm{C}_{20} \mathrm{H}_{21} \mathrm{~N}_{2} \mathrm{O}_{4}$ PS (416.43): C, 57.69; $\mathrm{H}, 5.08 ; \mathrm{N} 6.73 \%$. Found: $\mathrm{C}, 57.59 ; \mathrm{H}, 5.11 ; \mathrm{N} 6.78 \%$.

Diethyl (4,8-dimethyl-2-oxo-3,4-dihydro-2H-benzo[4,5]thiazolo[3,2-a]pyrimidin-3-yl)phosphonate (12f). (71\%); yellow crystals; mp 152-154 ${ }^{\circ} \mathrm{C}$; IR v(cm $\left.{ }^{-1}\right): 2983(\mathrm{w}), 1664$ (m), 1498 (vs), 1357 (m), 1247 (m), 1019 (s); ${ }^{1} \mathrm{H}$ NMR $\left(250 \mathrm{MHz}, \mathrm{CDCl}_{3}\right) \delta 7.39$ (s, 1H, H-Ar), 7.26 (d, J $\left.8.2 \mathrm{~Hz}, 1 \mathrm{H}, H-\mathrm{Ar}\right), 7.12$ (d, J $\left.8.2 \mathrm{~Hz}, 1 \mathrm{H}, H-\mathrm{Ar}\right), 5.03$ 
(dqd, J 14.0, 6.9, $0.6 \mathrm{~Hz}, 1 \mathrm{H}, \mathrm{H}-4), 4.22-4.15\left(\mathrm{~m}, 2 \mathrm{H}, \mathrm{CH}_{2} \mathrm{OP}\right), 3.97-3.89\left(\mathrm{~m}, 2 \mathrm{H}, \mathrm{CH}_{2} \mathrm{OP}\right), 3.15$ (dd, J $23.6,0.6$ $\mathrm{Hz}, 1 \mathrm{H}, \mathrm{H}-3$ ), $2.43\left(\mathrm{~s}, 3 \mathrm{H}, \mathrm{CH}_{3}\right), 1.48$ (dd, J 6.8, $1.5 \mathrm{~Hz}, 3 \mathrm{H}, \mathrm{CH}_{3} \mathrm{CH}$ ), 1.34 (t, J 7.1 Hz, 3H, $\mathrm{CH}_{3} \mathrm{CH}_{2} \mathrm{O}$ ), 1.00 (t, J 7.0 $\mathrm{Hz}, 3 \mathrm{H}, \mathrm{CH}_{3} \mathrm{CH}_{2} \mathrm{O}$ ); ${ }^{13} \mathrm{C}$ NMR (176 MHz, CDCl 3 ) $\delta 171.5$ (s, C-Ar), 168.1 (d, J 4.6 Hz, C(O)), 135.1 (s, C-Ar), 134.4 (s, C-Ar), 128.3 (s, CH-Ar), 123.5 (s, C-Ar), 122.8 (s, CH-Ar), 110.3 (s, CH-Ar), 63.4 (d, J 6.5 Hz, CH ${ }_{2} \mathrm{OP}$ ), 62.7 (d, J 7.2 $\mathrm{Hz}, \mathrm{CH}_{2} \mathrm{OP}$ ), 49.3 (d, J $6.0 \mathrm{~Hz}, \mathrm{C}-4$ ), 45.3 (d, J $126.9 \mathrm{~Hz}, \mathrm{C}-3$ ), 20.9 (s, $\mathrm{CH}_{3}$ ), 18.9 (d, J $17.8 \mathrm{~Hz}, \mathrm{CH}_{3}$ ), 16.0 (d, J 6.2 $\mathrm{Hz}, \mathrm{CH}_{3} \mathrm{CH}_{2}$ ), 15.8 (d, J $6.3 \mathrm{~Hz}, \mathrm{CH}_{3} \mathrm{CH}_{2}$ ); ${ }^{31} \mathrm{P} \mathrm{NMR}\left(283 \mathrm{MHz}, \mathrm{CDCl}_{3}\right) \delta$ 19.13. Anal. Calcd for $\mathrm{C}_{16} \mathrm{H}_{21} \mathrm{~N}_{2} \mathrm{O}_{4} \mathrm{PS}$ (368.39): C, 52.17; H, 5.75; N 7.60\%. Found: C, 52.11; H, 5.80; N 7.63\%.

Diethyl (4-ethyl-8-methyl-2-oxo-3,4-dihydro-2H-benzo[4,5]thiazolo[3,2-a]pyrimidin-3-yl)phosphonate (12g). (85\%); yellow crystals; mp 120-122 ${ }^{\circ} \mathrm{C}$; IR v(cm ${ }^{-1}$ ): 2935 (w), 1661 (s), 1492 (vs), 1348 (s), 1238 (s), 1015 (vs); ${ }^{1} \mathrm{H}$ NMR (700 MHz, CDCl $) \delta 7.38$ (s, 1H, H-Ar), 7.25 (d, J $8.3 \mathrm{~Hz}, 1 \mathrm{H}, H$-Ar), 7.12 (d, J $8.3 \mathrm{~Hz}, 1 \mathrm{H}, H-A r), 4.82$ (dtd, J 15.4, 6.9, $0.6 \mathrm{~Hz}, 1 \mathrm{H}, \mathrm{H}-4), 4.22-4.16\left(\mathrm{~m}, 2 \mathrm{H}, \mathrm{CH}_{2} \mathrm{OP}\right.$ ), $3.95-3.89\left(\mathrm{~m}, 2 \mathrm{H}, \mathrm{CH}_{2} \mathrm{OP}\right), 3.25$ (dd, J 24.0, $0.6 \mathrm{~Hz}, 1 \mathrm{H}$, $\mathrm{H}-3), 2.43\left(\mathrm{~s}, 3 \mathrm{H}, \mathrm{CH}_{3}\right), 1.91-1.82\left(\mathrm{~m}, 2 \mathrm{H}, \mathrm{CH}_{3} \mathrm{CH}_{2}\right), 1.34\left(\mathrm{~d}, J 7.1 \mathrm{~Hz}, 3 \mathrm{H}, \mathrm{CH}_{3} \mathrm{CH}_{2} \mathrm{O}\right), 1.01(\mathrm{t}, J 7.5 \mathrm{~Hz}, 3 \mathrm{H}$, $\mathrm{CH}_{3} \mathrm{CH}$ ), $0.98\left(\mathrm{t}, J 7.1 \mathrm{~Hz}, 3 \mathrm{H}, \mathrm{CH}_{3} \mathrm{CH}_{2} \mathrm{O}\right) ;{ }^{13} \mathrm{C} \mathrm{NMR}\left(176 \mathrm{MHz}, \mathrm{CDCl}_{3}\right) \delta 172.0(\mathrm{~s}, \mathrm{C}-\mathrm{Ar}), 168.2(\mathrm{~d}, J 6.5 \mathrm{~Hz}, \mathrm{C}(\mathrm{O})$ ), 135.6 (s, C-Ar), 134.3 (s, C-Ar), 128.2 (s, CH-Ar), 123.4 (s, C-Ar), 122.7 (s, CH-Ar), 110.7 (s, CH-Ar), 63.4 (d, J 6.5 $\mathrm{Hz}, \mathrm{CH}_{2} \mathrm{OP}$ ), 62.7 (d, J 7.2 Hz, CH $\mathrm{C}_{2} \mathrm{OP}$ ), 54.6 (d, J 3.6 Hz, C-4), 42.7 (d, J 126.7 Hz, C-3), 26.2 (d, J 16.5 Hz, CH $\mathrm{CH}_{2}$ ), 20.9 (s, $\mathrm{CH}_{3}$ ), 16.1 (d, J $6.2 \mathrm{~Hz}, \mathrm{CH}_{3} \mathrm{CH}_{2}$ ), 15.8 (d, J $6.2 \mathrm{~Hz}, \mathrm{CH}_{3} \mathrm{CH}_{2}$ ), 9.5 (s, $\left.\mathrm{CH}_{3}\right) ;{ }^{31} \mathrm{P} \mathrm{NMR}(283 \mathrm{MHz}, \mathrm{CDCl}$ ) $\delta$ 19.65. Anal. Calcd for $\mathrm{C}_{17} \mathrm{H}_{23} \mathrm{~N}_{2} \mathrm{O}_{4} \mathrm{PS}$ (382.41): C, 53.39; $\mathrm{H}, 6.06 ; \mathrm{N} 7.33 \%$. Found: $\mathrm{C}, 53.29 ; \mathrm{H}, 6.07 ; \mathrm{N} 7.35 \%$.

Diethyl (4-isopropyl-8-methyl-2-oxo-3,4-dihydro-2H-benzo[4,5]thiazolo[3,2-a]pyrimidin-3-yl)phosphonate (12h). (78\%); yellow crystals; mp 168-170 ${ }^{\circ} \mathrm{C}$; IR v(cm $\left.{ }^{-1}\right)$ : 2941 (w), 1663 (m), 1490 (vs), 1337 (m), 1237 (s), 1012 (vs); ${ }^{1} \mathrm{H}$ NMR (250 MHz, CDCl $) \delta 7.33$ (s, $1 \mathrm{H}, H$-Ar), 7.18 (d, J $8.4 \mathrm{~Hz}, 1 \mathrm{H}, H-\mathrm{Ar}$ ), 7.04 (d, J $\left.8.4 \mathrm{~Hz}, 1 \mathrm{H}, H-\mathrm{Ar}\right), 4.63$ (ddd, J 17.3, 5.4, 0.7 Hz, 1H, H-4), $4.20-4.03\left(\mathrm{~m}, 2 \mathrm{H}, \mathrm{CH}_{2} \mathrm{OP}\right), 3.93-3.77$ (m, 2H, CH $\left.\mathrm{H}_{2} \mathrm{OP}\right), 3.23$ (dd, J 24.9, 0.7 $\mathrm{Hz}, 1 \mathrm{H}, \mathrm{H}-3), 2.35\left(\mathrm{~s}, 3 \mathrm{H}, \mathrm{CH}_{3}\right), 2.32-2.22\left(\mathrm{~m}, 1 \mathrm{H},\left(\mathrm{CH}_{3}\right)_{2} \mathrm{CH}\right), 1.24\left(\mathrm{~d}, J 7.1 \mathrm{~Hz}, 3 \mathrm{H}, \mathrm{CH}_{3} \mathrm{CH}_{2} \mathrm{O}\right), 0.95(\mathrm{~d}, J 7.0 \mathrm{~Hz}$, $\left.3 \mathrm{H}, \mathrm{CH}_{3} \mathrm{CH}\right), 0.91\left(\mathrm{~d}, J 7.1 \mathrm{~Hz}, 3 \mathrm{H}, \mathrm{CH}_{3} \mathrm{CH}\right), 0.87\left(\mathrm{t}, J 7.0 \mathrm{~Hz}, 3 \mathrm{H}, \mathrm{CH}_{3} \mathrm{CH}_{2} \mathrm{O}\right) ;{ }^{13} \mathrm{C} \mathrm{NMR}\left(176 \mathrm{MHz}, \mathrm{CDCl}_{3}\right) \delta 172.6(\mathrm{~s}$, C-Ar), 168.7 (d, J 5.0 Hz, C(O)), 136.0 (s, C-Ar), 134.2 (s, C-Ar), 128.0 (s, CH-Ar), 123.2 (s, C-Ar), 122.6 (s, CH-Ar), 111.1 (s, CH-Ar), 63.3 (d, J $6.6 \mathrm{~Hz}, \mathrm{CH}_{2} \mathrm{OP}$ ), 62.7 (d, J $7.0 \mathrm{~Hz}, \mathrm{CH}_{2} \mathrm{OP}$ ), 58.6 (d, J $4.1 \mathrm{~Hz}, \mathrm{C}-4$ ), 39.7 (d, J $127.3 \mathrm{~Hz}$, C-3), 31.6 (d, J $15.1 \mathrm{~Hz}, \mathrm{CH}_{3} \mathrm{CH}$ ), 20.9 (s, $\mathrm{CH}_{3}$ ), 18.6 (s, $\left.\mathrm{CH}_{3}\right), 17.0$ (s, $\mathrm{CH}_{3}$ ), 16.0 (d, J $6.1 \mathrm{~Hz}, \mathrm{CH}_{3} \mathrm{CH}_{2}$ ), 15.8 (d, J 6.4 $\mathrm{Hz}, \mathrm{CH}_{3} \mathrm{CH}_{2}$ ); ${ }^{31} \mathrm{P}$ NMR (283 MHz, $\mathrm{CDCl}_{3}$ ) $\delta$ 19.76. Anal. Calcd for $\mathrm{C}_{18} \mathrm{H}_{25} \mathrm{~N}_{2} \mathrm{O}_{4} \mathrm{PS}$ (396.44): C, 54.53; $\mathrm{H}, 6.36 ; \mathrm{N}$ 7.07\%. Found: C, 54.45; $\mathrm{H}, 6.39 ; \mathrm{N} 7.12 \%$.

Diethyl (4-butyl-8-methyl-2-oxo-3,4-dihydro-2H-benzo[4,5]thiazolo[3,2-a]pyrimidin-3-yl)phosphonate (12i). (74\%); yellow crystals; mp 164-166 ${ }^{\circ} \mathrm{C}$; IR v(cm $\left.{ }^{-1}\right)$ : 2927 (w), 1661 (m), 1494 (vs), 1343 (m), 1242 (s), 1015 (s); ${ }^{1} \mathrm{H}$ NMR (700 MHz, CDCl $) \delta 7.38$ (s, 1H, H-Ar), 7.25 (d, J $8.3 \mathrm{~Hz}, 1 \mathrm{H}, H-\mathrm{Ar}$ ), 7.11 (d, J 8.3 Hz, 1H, H-Ar), 4.86 (dtd, J 14.7, 6.9, $0.5 \mathrm{~Hz}, 1 \mathrm{H}, \mathrm{H}-4), 4.22-4.15\left(\mathrm{~m}, 2 \mathrm{H}, \mathrm{CH}_{2} \mathrm{OP}\right), 3.95-3.88\left(\mathrm{~m}, 2 \mathrm{H}, \mathrm{CH}_{2} \mathrm{OP}\right), 3.26$ (dd, J $24.2,0.5 \mathrm{~Hz}$, $1 \mathrm{H}, \mathrm{H}-3), 2.43\left(\mathrm{~s}, 3 \mathrm{H}, \mathrm{CH}_{3}\right), 1.84-1.79\left(\mathrm{~m}, 2 \mathrm{H}, \mathrm{CH}_{2}\right), 1.44-1.35\left(\mathrm{~m}, 4 \mathrm{H}, 2 \times \mathrm{CH}_{2}\right), 1.34(\mathrm{~d}, J 7.3 \mathrm{~Hz}, 3 \mathrm{H}$, $\left.\mathrm{CH}_{3} \mathrm{CH}_{2} \mathrm{O}\right), 0.98\left(\mathrm{~d}, J 7.1 \mathrm{~Hz}, 3 \mathrm{H}, \mathrm{CH}_{3} \mathrm{CH}_{2} \mathrm{O}\right), 0.88\left(\mathrm{~d}, J 7.1 \mathrm{~Hz}, 3 \mathrm{H}, \mathrm{CH}_{3} \mathrm{CH}\right) ;{ }^{13} \mathrm{C} \mathrm{NMR}\left(176 \mathrm{MHz}, \mathrm{CDCl}_{3}\right) \delta 171.9(\mathrm{~s}$, C-Ar), 168.2 (d, J $4.5 \mathrm{~Hz}, C(\mathrm{O})$ ), 135.6 (s, C-Ar), 134.3 (s, C-Ar), 128.2 (s, CH-Ar), 123.4 (s, C-Ar), 122.7 (s, CH-Ar), 110.6 (s, CH-Ar), 63.3 (d, J $6.7 \mathrm{~Hz}, \mathrm{CH}_{2} \mathrm{OP}$ ), 62.7 (d, J $7.2 \mathrm{~Hz}, \mathrm{CH}_{2} \mathrm{OP}$ ), 53.5 (d, J $5.5 \mathrm{~Hz}, \mathrm{C}-4$ ), 42.9 (d, J $126.7 \mathrm{~Hz}$, C-3), 32.6 (d, J $16.0 \mathrm{~Hz}, \mathrm{CH}_{2} \mathrm{CH}_{2}$ ), 26.9 (s, $\mathrm{CH}_{2} \mathrm{CH}_{2}$ ), 22.1 (s, $\mathrm{CH}_{2} \mathrm{CH}_{2}$ ), 20.9 (s, $\mathrm{CH}_{3}$ ), 16.0 (d, J 6.2 Hz, CH${ }_{3} \mathrm{CH}_{2} \mathrm{O}$ ), $15.8\left(\mathrm{~d}, J 5.8 \mathrm{~Hz}, \mathrm{CH}_{3} \mathrm{CH}_{2} \mathrm{O}\right.$ ), $13.5\left(\mathrm{~s}, \mathrm{CH}_{3} \mathrm{CH}_{2}\right) ;{ }^{31} \mathrm{P} \mathrm{NMR}\left(283 \mathrm{MHz}, \mathrm{CDCl}_{3}\right) \delta$ 19.61. Anal. Calcd for $\mathrm{C}_{19} \mathrm{H}_{27} \mathrm{~N}_{2} \mathrm{O}_{4} \mathrm{PS}$ (410.47): C, 55.60; H, 6.63; N 6.82\%. Found: C, 55.47; H, 6.66; N 6.86\%.

Diethyl (8-methyl-2-oxo-4-phenyl-3,4-dihydro-2H-benzo[4,5]thiazolo[3,2-a]pyrimidin-3-yl)phosphonate (12j). (79\%); yellow crystals; mp 180-182 ${ }^{\circ} \mathrm{C}$; IR v(cm $\left.{ }^{-1}\right): 2982$ (w), 1670 (m), 1499 (vs), 1351 (m), 1245 (m), 1017 (s); ${ }^{1} \mathrm{H}$ NMR (700 MHz, CDCl $) \delta 7.39$ (s, $1 \mathrm{H}, H$-Ar), $7.35-7.28$ (m, 4H, H-Ar), $7.22-7.18$ (m, 2H, H-Ar), $7.13(\mathrm{~d}, J 8.4 \mathrm{~Hz}, 1 \mathrm{H}, H-\mathrm{Ar}), 6.97-6.94(\mathrm{~m}, 1 \mathrm{H}, H-\mathrm{Ar}), 5.93$ (dd, J 17.3, $0.7 \mathrm{~Hz}, 1 \mathrm{H}, H-4), 4.26-4.18(\mathrm{~m}, 2 \mathrm{H}$, 
$\mathrm{CH}_{2} \mathrm{OP}$ ), $4.05-3.98\left(\mathrm{~m}, 2 \mathrm{H}, \mathrm{CH}_{2} \mathrm{OP}\right), 3.39$ (dd, J 23.4, $\left.0.7 \mathrm{~Hz}, 1 \mathrm{H}, \mathrm{H}-3\right), 2.38\left(\mathrm{~s}, 3 \mathrm{H}, \mathrm{CH}_{3}\right), 1.36(\mathrm{~d}, J 7.3 \mathrm{~Hz}, 3 \mathrm{H}$, $\mathrm{CH}_{3} \mathrm{CH}_{2} \mathrm{O}$ ), 1.07 (d, J $\left.7.1 \mathrm{~Hz}, 3 \mathrm{H}, \mathrm{CH}_{3} \mathrm{CH}_{2} \mathrm{O}\right) ;{ }^{13} \mathrm{C} \mathrm{NMR}\left(176 \mathrm{MHz}, \mathrm{CDCl}_{3}\right) \delta 172.4$ (s, C-Ar), 166.9 (d, J $4.5 \mathrm{~Hz}, \mathrm{C}(\mathrm{O})$ ), 136.2 (d, J 16.2 Hz, C-Ar), 134.9 (s, CH-Ar), 134.3 (s, CH-Ar), 129.1 (s, 2 x CH-Ar), 128.6 (s, C-Ar), 127.9 (s, C-Ar), 124.7 (s, CH-Ar), 122.5 (s, $2 \times \mathrm{CH}-\mathrm{Ar}$ ), 122.4 (s, CH-Ar), 110.6 (s, CH-Ar), 63.1 (d, J 6.6 Hz, CH ${ }_{2} \mathrm{OP}$ ), 62.6 (d, J 7.1 $\mathrm{Hz}, \mathrm{CH}_{2} \mathrm{OP}$ ), 56.1 (d, J $\left.7.1 \mathrm{~Hz}, \mathrm{C}-4\right), 46.6$ (d, J $\left.123.1 \mathrm{~Hz}, \mathrm{C}-3\right), 20.5$ (s, $\mathrm{CH}_{3}$ ), 15.7 (d, J 3.8 Hz, CH $\left.\mathrm{CH}_{2} \mathrm{O}\right), 15.5$ (d, J $\left.5.5 \mathrm{~Hz}, \mathrm{CH}_{3} \mathrm{CH}_{2} \mathrm{O}\right) ;{ }^{31} \mathrm{P} \mathrm{NMR}\left(283 \mathrm{MHz}, \mathrm{CDCl}_{3}\right) \delta$ 18.90. Anal. Calcd for $\mathrm{C}_{21} \mathrm{H}_{23} \mathrm{~N}_{2} \mathrm{O}_{4} \mathrm{PS}(430.46): \mathrm{C}, 58.60 ; \mathrm{H}, 5.39$; N 6.51\%. Found: C, 58.43; $H, 5.41 ;$ N 6.54\%.

\section{Diethyl (4-butyl-8-methoxy-2-oxo-3,4-dihydro-2H-benzo[4,5]thiazolo[3,2-a]pyrimidin-3-yl)phosphonate}

(12m). (89\%); yellow crystals; mp 148-150 ${ }^{\circ} \mathrm{C} ; \mathrm{IR} \mathrm{v}\left(\mathrm{cm}^{-1}\right)$ : 2925 (w), 1658 (m), 1496 (vs), 1360 (m), 1238 (s), 1014 (s); ${ }^{1} \mathrm{H}$ NMR (700 MHz, CDCl $) \delta 7.13$ (d, J $8.9 \mathrm{~Hz}, 1 \mathrm{H}, \mathrm{H}-\mathrm{Ar}$ ), 7.10 (d, J $2.5 \mathrm{~Hz}, 1 \mathrm{H}, \mathrm{H}-\mathrm{Ar}$ ), 7.01 (dd, J 8.9, 2.5 $\mathrm{Hz}, 1 \mathrm{H}, \mathrm{H}-\mathrm{Ar}$ ), 4.84 (dtd, J 15.4, 6.9, $0.6 \mathrm{~Hz}, 1 \mathrm{H}, \mathrm{H}-4), 4.22-4.15\left(\mathrm{~m}, 2 \mathrm{H}, \mathrm{CH}_{2} \mathrm{OP}\right), 3.97-3.89\left(\mathrm{~m}, 2 \mathrm{H}, \mathrm{CH} \mathrm{OP}_{2}\right.$, $3.85\left(\mathrm{~s}, 3 \mathrm{H}, \mathrm{CH}_{3} \mathrm{O}\right), 3.25(\mathrm{dd}, J 24.1,0.6 \mathrm{~Hz}, 1 \mathrm{H}, \mathrm{H}-3), 1.85-1.79\left(\mathrm{~m}, 2 \mathrm{H}, \mathrm{CH}_{2}\right), 1.42-1.36\left(\mathrm{~m}, 2 \mathrm{H}, \mathrm{CH}_{2}\right), 1.34(\mathrm{~d}$, J $\left.7.1 \mathrm{~Hz}, 3 \mathrm{H}, \mathrm{CH}_{3} \mathrm{CH}_{2} \mathrm{O}\right), 1.33-1.28\left(\mathrm{~m}, 2 \mathrm{H}, \mathrm{CH}_{2}\right), 1.00\left(\mathrm{~d}, J 7.1 \mathrm{~Hz}, 3 \mathrm{H}, \mathrm{CH}_{3} \mathrm{CH}_{2} \mathrm{O}\right), 0.88(\mathrm{~d}, J 7.1 \mathrm{~Hz}, 3 \mathrm{H}, \mathrm{CH} \mathrm{CH}$ ); ${ }^{13} \mathrm{C} \mathrm{NMR}\left(176 \mathrm{MHz}, \mathrm{CDCl}_{3}\right.$ ) $\delta 171.3$ (s, C-Ar), 167.7 (d, J $4.4 \mathrm{~Hz}, \mathrm{C}(\mathrm{O})$ )), 156.4 (s, C-Ar), 131.3 (s, CH-Ar), 124.4 (s, CH-Ar), 114.1 (s, CH-Ar), 111.4 (s, C-Ar), 107.8 (s, C-Ar), 62.9 (d, J $6.6 \mathrm{~Hz}, \mathrm{CH}_{2} \mathrm{OP}$ ), 62.4 (d, J 7.3 Hz, CH $\mathrm{H}_{2} \mathrm{OP}$ ), 55.5 (s, $\mathrm{CH}_{3} \mathrm{O}$ ), 53.3 (d, J $5.1 \mathrm{~Hz}, \mathrm{C}-4$ ), 42.7 (d, J $126.9 \mathrm{~Hz}, \mathrm{C}-3$ ), 32.3 (d, J $16.1 \mathrm{~Hz}, \mathrm{CH}_{2} \mathrm{CH}$ ), $26.6\left(\mathrm{~s}, \mathrm{CH}_{2} \mathrm{CH}_{2}\right), 21.8(\mathrm{~s}$, $\mathrm{CH}_{2} \mathrm{CH}_{2}$ ), 15.8 (d, J $5.8 \mathrm{~Hz}, \mathrm{CH}_{3} \mathrm{CH}_{2} \mathrm{O}$ ), 15.6 (d, J $\left.6.0 \mathrm{~Hz}, \mathrm{CH}_{3} \mathrm{CH}_{2} \mathrm{O}\right), 13.3\left(\mathrm{~s}, \mathrm{CH}_{3} \mathrm{CH}_{2}\right.$ ); ${ }^{31} \mathrm{P} \mathrm{NMR}(283 \mathrm{MHz}, \mathrm{CDCl}) \delta$ 19.67. Anal. Calcd for $\mathrm{C}_{19} \mathrm{H}_{27} \mathrm{~N}_{2} \mathrm{O}_{5} \mathrm{PS}$ (426.47): C, 53.51; $\mathrm{H}, 6.38 ; \mathrm{N} 6.57 \%$. Found: $\mathrm{C}, 53.38 ; \mathrm{H}, 6.40 ; \mathrm{N} 6.61 \%$.

General procedure for the synthesis of 3-methylene-3,4-dihydro-2H-benzothiazolopyrimidin-2-one 13a-j,m. To a solution of the corresponding diethyl (2-oxo-3,4-dihydro-2H-benzothiazolopyrimidin-3-yl)phosphonate $12 \mathrm{a}-\mathrm{j}, \mathrm{m}(0.5 \mathrm{mmol})$ in THF $(5 \mathrm{~mL}), \mathrm{K}_{2} \mathrm{CO}_{3}(138 \mathrm{mg}, 1.0 \mathrm{mmol})$ was added and the resulting mixture was stirred at $\mathrm{rt}$ for $30 \mathrm{~min}$. Then, paraformaldehyde $(75 \mathrm{mg}, 2.5 \mathrm{mmol})$ was added in one portion. After $24 \mathrm{~h}$, the reaction mixture was quenched with brine $(10 \mathrm{~mL})$ and extracted with $\mathrm{CH}_{2} \mathrm{Cl}_{2}(3 \times 10 \mathrm{~mL})$. The organic layer was dried over $\mathrm{MgSO}_{4}$ and the solvent was evaporated. The crude product was purified by column chromatography (eluent: $\mathrm{Et}_{2} \mathrm{O}$ ).

4-Methyl-3-methylene-3,4-dihydro-2H-benzo[4,5]thiazolo[3,2-a]pyrimidin-2-one (13a). (76\%); yellow oil; IR

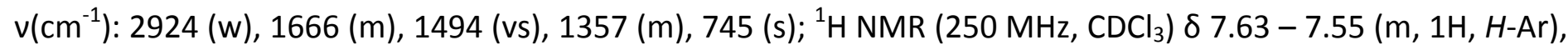
$7.51-7.41(\mathrm{~m}, 1 \mathrm{H}, \mathrm{H}-\mathrm{Ar}), 7.35-7.27(\mathrm{~m}, 1 \mathrm{H}, \mathrm{H}-\mathrm{Ar}), 7.24-7.16(\mathrm{~m}, 1 \mathrm{H}, \mathrm{H}-\mathrm{Ar}), 6.38(\mathrm{~s}, 1 \mathrm{H}, \mathrm{CH}=\mathrm{C}), 5.60(\mathrm{~s}, 1 \mathrm{H}$, $\left.\mathrm{CH}_{2}=\mathrm{C}\right), 5.21(\mathrm{q}, J 6.8 \mathrm{~Hz}, 1 \mathrm{H}, \mathrm{H}-4), 1.56\left(\mathrm{~d}, J 6.8 \mathrm{~Hz}, 3 \mathrm{H}, \mathrm{CH}_{3}\right) ;{ }^{13} \mathrm{C} \mathrm{NMR}\left(63 \mathrm{MHz}, \mathrm{CDCl}_{3}\right) \delta 171.6(\mathrm{~s}, \mathrm{C}-\mathrm{Ar}), 167.1$ (s, C(O)), 137.3 (s, C=), 135.6 (s, C-Ar), 127.3 (s, CH-Ar), 124.3 (s, C-Ar), 124.2 (s, CH-Ar), 123.7 (s, CH-Ar), 122.8 (s, $\mathrm{CH}_{2}=$ ), 110.8 (s, CH-Ar), 54.7 (s, C-2), 21.6 (s, $\mathrm{CH}_{3}$ ). Anal. Calcd for $\mathrm{C}_{12} \mathrm{H}_{10} \mathrm{~N}_{2} \mathrm{OS}$ (230.29): C, 62.59; $\mathrm{H}, 4.38 ; \mathrm{N}$, $12.16 \%$. Found: $\mathrm{C}, 62.45 ; \mathrm{H}, 4.41 ; \mathrm{N}, 12.19 \%$.

4-Ethyl-3-methylene-3,4-dihydro-2H-benzo[4,5]thiazolo[3,2-a]pyrimidin-2-one (13b). (82\%); yellow oil; IR $\mathrm{v}\left(\mathrm{cm}^{-1}\right)$ : 2953 (w), 1635 (m), 1496 (vs), 1342 (m), 746 (s); ${ }^{1} \mathrm{H}$ NMR (700 MHz, CDCl $) \delta 7.58(\mathrm{~d}, J 7.9 \mathrm{~Hz}, 1 \mathrm{H}, \mathrm{H}-$ Ar), 7.45 (d, J $7.9 \mathrm{~Hz}, 1 \mathrm{H}, H-\mathrm{Ar}$ ), 7.28 (d, J $7.9 \mathrm{~Hz}, 1 \mathrm{H}, \mathrm{H}-\mathrm{Ar}$ ), 7.16 (d, J 7.9 Hz, 1H, H-Ar), $6.43\left(\mathrm{~s}, 1 \mathrm{H}, \mathrm{CH}_{2}=\mathrm{C}\right), 5.55$ $\left(\mathrm{s}, 1 \mathrm{H}, \mathrm{CH}_{2}=\mathrm{C}\right), 4.97(\mathrm{dd}, J$ 8.5, 3.9 Hz, $1 \mathrm{H}, \mathrm{H}-4), 1.92-1.87\left(\mathrm{~m}, 2 \mathrm{H}, \mathrm{CH}_{3} \mathrm{CH}_{2}\right), 0.96\left(\mathrm{t}, J \mathrm{~J} .4 \mathrm{~Hz}, 3 \mathrm{H}, \mathrm{CH}_{3}\right) ;{ }^{13} \mathrm{C} \mathrm{NMR}$ $\left(176 \mathrm{MHz}, \mathrm{CDCl}_{3}\right.$ ) $\delta 172.2$ (s, C-Ar), 167.6 (s, C(O)), 137.6 (s, C=), 135.5 (s, C-Ar), 127.2 (s, CH-Ar), 125.3 (s, CHAr), 124.2 (s, CH-Ar), 124.1 (s, C-Ar), 122.9 (s, $\mathrm{CH}_{2}=$ ), 110.8 (s, CH-Ar), 60.4 (s, C-2), 27.2 (s, $\mathrm{CH}_{3} \mathrm{CH}_{2}$ ), 8.5 (s, $\mathrm{CH}_{3} \mathrm{CH}_{2}$ ). Anal. Calcd for $\mathrm{C}_{13} \mathrm{H}_{12} \mathrm{~N}_{2} \mathrm{OS}$ (244.31): C, 63.91; $\mathrm{H}, 4.95 ; \mathrm{N}, 11.47 \%$. Found: $\mathrm{C}, 63.90 ; \mathrm{H}, 4.99 ; \mathrm{N}, 11.50 \%$. 4-Isopropyl-3-methylene-3,4-dihydro-2H-benzo[4,5]thiazolo[3,2-a]pyrimidin-2-one (13c). (32\%); yellow oil; IR v(cm ${ }^{-1}$ ): 2954 (w), 1675 (s), 1491 (vs), 1337 (s), 1240 (m), 750 (m); $\left.{ }^{1} \mathrm{H} \mathrm{NMR} \mathrm{(700} \mathrm{MHz,} \mathrm{CDCl}\right)$ ) 7.58 (d, J 7.9 $\mathrm{Hz}, 1 \mathrm{H}, H-\mathrm{Ar}$ ), 7.43 (d, J $7.9 \mathrm{~Hz}, 1 \mathrm{H}, H-\mathrm{Ar}), 7.28$ (d, J $7.9 \mathrm{~Hz}, 1 \mathrm{H}, H-\mathrm{Ar}$ ), 7.12 (d, J $7.9 \mathrm{~Hz}, 1 \mathrm{H}, H-\mathrm{Ar}), 6.47$ (s, $1 \mathrm{H}$, $\mathrm{CH}_{2}=\mathrm{C}$ ), $5.50\left(\mathrm{~s}, 1 \mathrm{H}, \mathrm{CH}_{2}=\mathrm{C}\right.$ ), $4.89(\mathrm{~d}, J 4.0 \mathrm{~Hz}, 1 \mathrm{H}, \mathrm{H}-4), 2.39$ (heptd, J 6.9, 4.0,1H, $\left.\mathrm{CH}\left(\mathrm{CH}_{3}\right)_{2}\right), 1.03(\mathrm{~d}, J 6.9 \mathrm{~Hz}$, 
$3 \mathrm{H}, \mathrm{CH}_{3}$ ), 0.88 (d, J $\left.6.9 \mathrm{~Hz}, 3 \mathrm{H}, \mathrm{CH}_{3}\right) ;{ }^{13} \mathrm{C} \mathrm{NMR}\left(176 \mathrm{MHz}, \mathrm{CDCl}_{3}\right) \delta 172.5$ (s, C-Ar), 168.2 (s, C(O)), 137.8 (s, C=), 131.1 (s, C-Ar), 127.1 (s, CH-Ar), 126.5 (s, CH-Ar), 124.2 (s, CH-Ar), 123.9 (s, C-Ar), 122.9 (s, CH $=$ ), 111.2 (s, CHAr), 64.4 (s, C-2), 31.9 (s, $\mathrm{CH}_{3} \mathrm{CH}$ ), 18.2 (s, $\mathrm{CH}_{3} \mathrm{CH}$ ), 16.0 (s, $\mathrm{CH}_{3} \mathrm{CH}$ ). Anal. Calcd for $\mathrm{C}_{14} \mathrm{H}_{14} \mathrm{~N}_{2} \mathrm{OS}$ (258.34): C, $65.09 ; \mathrm{H}, 5.46 ; \mathrm{N}, 10.84 \%$. Found: $\mathrm{C}, 65.02 ; \mathrm{H}, 5.48 ; \mathrm{N}, 10.88 \%$.

4-Butyl-3-methylene-3,4-dihydro-2H-benzo[4,5]thiazolo[3,2-a]pyrimidin-2-one (13d). (87\%); yellow oil; IR $\mathrm{v}\left(\mathrm{cm}^{-1}\right)$ : 2951 (w), 1669 (m), 1497 (vs), 1359 (s), 1242 (m), 751 (m); ${ }^{1} \mathrm{H} \mathrm{NMR} \mathrm{(700} \mathrm{MHz,} \mathrm{CDCl}{ }_{3}$ ) 7.58 (d, J 7.9 $\mathrm{Hz}, 1 \mathrm{H}, H$-Ar), 7.45 (d, J $7.9 \mathrm{~Hz}, 1 \mathrm{H}, H-\mathrm{Ar}), 7.27$ (d, J $7.9 \mathrm{~Hz}, 1 \mathrm{H}, H-\mathrm{Ar}), 7.16$ (d, J $7.9 \mathrm{~Hz}, 1 \mathrm{H}, H-\mathrm{Ar}), 6.40(\mathrm{~s}, 1 \mathrm{H}$, $\left.\mathrm{CH}_{2}=\mathrm{C}\right), 5.53\left(\mathrm{~s}, 1 \mathrm{H}, \mathrm{CH}_{2}=\mathrm{C}\right), 5.02(\mathrm{dd}, J 9.0,3.7 \mathrm{~Hz}, 1 \mathrm{H}, \mathrm{H}-4), 1.91-1.83\left(\mathrm{~m}, 1 \mathrm{H}, \mathrm{CH}_{2}\right), 1.82-1.75\left(\mathrm{~m}, 1 \mathrm{H}, \mathrm{CH}_{2}\right)$, $1.36-1.21\left(\mathrm{~m}, 4 \mathrm{H}, 2 \times \mathrm{CH}_{2}\right), 0.85\left(\mathrm{t}, J 7.0 \mathrm{~Hz}, 3 \mathrm{H}, \mathrm{CH}_{3}\right) ;{ }^{13} \mathrm{C} \mathrm{NMR}\left(176 \mathrm{MHz}, \mathrm{CDCl}_{3}\right) \delta 172.1$ (s, C-Ar), $167.6(\mathrm{~s}$, $C(\mathrm{O})$ ), 137.6 (s, C=), 133.9 (s, C-Ar), 127.2 (s, CH-Ar), 125.0 (s, CH-Ar), 124.2 (s, CH-Ar), 124.0 (s, C-Ar), 122.9 (s, $\mathrm{CH}_{2}=$ ), 110.8 (s, CH-Ar), 59.2 (s, C-2), 33.5 (s, $\mathrm{CH}_{2}$ ), 25.9 (s, $\mathrm{CH}_{2}$ ), 22.2 (s, $\mathrm{CH}_{2}$ ), 13.7 (s, CH$)_{3}$. Anal. Calcd for $\mathrm{C}_{15} \mathrm{H}_{16} \mathrm{~N}_{2} \mathrm{OS}$ (272.37): C, 66.15; H, 5.92; N, 10.29\%. Found: C, 66.03; H, 5.96; N, 10.25\%.

3-Methylene-4-phenyl-3,4-dihydro-2H-benzo[4,5]thiazolo[3,2-a]pyrimidin-2-one (13e). (61\%); yellow oil; IR v(cm $\left.{ }^{-1}\right): 2996$ (w), 1660 (m), 1486 (vs), 1358 (s), 1159 (m), 741 (s); $\left.{ }^{1} \mathrm{H} \mathrm{NMR} \mathrm{(700} \mathrm{MHz,} \mathrm{CDCl}{ }_{3}\right) \delta 7.56$ (d, J 7.8 Hz, $1 \mathrm{H}, H$-Ar), $7.34-7.29$ (m, 2H, H-Ar), $7.29-7.25$ (m, 2H, H-Ar), $7.24-7.20(\mathrm{~m}, 3 \mathrm{H}, H-\mathrm{Ar}), 6.98(\mathrm{~d}, J 7.9 \mathrm{~Hz}, 1 \mathrm{H}$, $H-A r), 6.40\left(\mathrm{~s}, 1 \mathrm{H}, \mathrm{CH}_{2}=\mathrm{C}\right), 6.10\left(\mathrm{~s}, 1 \mathrm{H}, \mathrm{CH}_{2}=\mathrm{C}\right), 5.72(\mathrm{~s}, 1 \mathrm{H}, \mathrm{H}-4) ;{ }^{13} \mathrm{C} \mathrm{NMR}\left(176 \mathrm{MHz}, \mathrm{CDCl}_{3}\right) \delta 172.8(\mathrm{~s}, \mathrm{C}-\mathrm{Ar})$, 166.6 (s, C(O)), 137.9 (s, C=), 137.7 (s, C-Ar), 134.7 (s, C-Ar), 129.7 (s, 2 x CH-Ar), 128.9 (s, CH-Ar), 127.2 (s, CHAr), 125.6 (s, CH-Ar), 125.4 (s, 2 x CH-Ar), 124.4 (s, CH-Ar), 123.6 (s, C-Ar), 122.7 (s, CH $=111.7$ (s, CH-Ar), 62.7 (s, C-2). Anal. Calcd for $\mathrm{C}_{17} \mathrm{H}_{12} \mathrm{~N}_{2} \mathrm{OS}$ (292.36): C, 69.84; H, 4.14; N, 9.58\%. Found: C, 69.76; H, 4.12; N, 9.56\%.

4,8-Dimethyl-3-methylene-3,4-dihydro-2H-benzo[4,5]thiazolo[3,2-a]pyrimidin-2-one (13f). (82\%); yellow oil; IR v(cm ${ }^{-1}$ ): 2963 (w), 1665 (m), 1493 (vs), 1351 (s), 1158 (s), 746 (m); $\left.{ }^{1} \mathrm{H} \mathrm{NMR} \mathrm{(700} \mathrm{MHz,} \mathrm{CDCl}\right) \delta 7.36$ (s, $1 \mathrm{H}, \mathrm{H}-$ Ar), 7.24 (d, J $8.2 \mathrm{~Hz}, 1 \mathrm{H}, H$-Ar), 7.09 (d, J $8.2 \mathrm{~Hz}, 1 \mathrm{H}, H-\mathrm{Ar}), 6.34$ (s, 1H, CH$=\mathrm{C}$ ), $5.57(\mathrm{~s}, 1 \mathrm{H}, \mathrm{CH}=\mathrm{C}), 5.19$ (q, J $6.8 \mathrm{~Hz}, 1 \mathrm{H}, \mathrm{H}-4), 2.40\left(\mathrm{~s}, 3 \mathrm{H}, \mathrm{CH}_{3}\right), 1.52\left(\mathrm{~d}, J 6.8 \mathrm{~Hz}, 3 \mathrm{H}, \mathrm{CH}_{3}\right) ;{ }^{13} \mathrm{C} \mathrm{NMR}\left(176 \mathrm{MHz}, \mathrm{CDCl}_{3}\right) \delta 171.6(\mathrm{~s}, \mathrm{C}-\mathrm{Ar}), 167.1$ (s, C(O)), 135.8 (s, C=), 135.3 (s, C-Ar), 134.5 (s, C-Ar), 128.2 (s, CH-Ar), 124.1 (s, CH-Ar), 123.9 (s, C-Ar), 123.0 (s, $\mathrm{CH}_{2}=$ ), 110.5 (s, CH-Ar), 54.9 (s, C-2), 21.7 (s, $\mathrm{CH}_{3}$ ), 21.1 (s, $\mathrm{CH}_{3}$ ). Anal. Calcd for $\mathrm{C}_{13} \mathrm{H}_{12} \mathrm{~N}_{2} \mathrm{OS}$ (244.31): C, 63.91; $\mathrm{H}, 4.95 ; \mathrm{N}, 11.47 \%$. Found: $\mathrm{C}, 63.83 ; \mathrm{H}, 4.99 ; \mathrm{N}, 11.46 \%$.

4-Ethyl-8-methyl-3-methylene-3,4-dihydro-2H-benzo[4,5]thiazolo[3,2-a]pyrimidin-2-one (13g). (84\%); yellow oil; IR v(cm ${ }^{-1}$ ): 2962 (w), 1658 (m), 1487 (vs), 1354 (s), 1167 (s), 780 (m); $\left.{ }^{1} \mathrm{H} \mathrm{NMR} \mathrm{(700} \mathrm{MHz,} \mathrm{CDCl}{ }_{3}\right) \delta .34(\mathrm{~s}$, $1 \mathrm{H}, H$-Ar), 7.21 (d, J $8.3 \mathrm{~Hz}, 1 \mathrm{H}, \mathrm{H}$-Ar), 7.05 (d, J $8.3 \mathrm{~Hz}, 1 \mathrm{H}, \mathrm{H}-\mathrm{Ar}), 6.38\left(\mathrm{~s}, 1 \mathrm{H}, \mathrm{CH}_{2}=\mathrm{C}\right), 5.52\left(\mathrm{~s}, 1 \mathrm{H}, \mathrm{CH}_{2}=\mathrm{C}\right), 4.95$ (dd, J 8.4, $4.0 \mathrm{~Hz}, 1 \mathrm{H}, \mathrm{H}-4), 2.38\left(\mathrm{~s}, 3 \mathrm{H}, \mathrm{CH}_{3}\right), 1.90-1.78\left(\mathrm{~m}, 2 \mathrm{H}, \mathrm{CH}_{3} \mathrm{CH}_{2}\right), 0.90\left(\mathrm{t}, J 7.4 \mathrm{~Hz}, 3 \mathrm{H}, \mathrm{CH}_{3}\right) ;{ }^{13} \mathrm{C} \mathrm{NMR}$ (176 MHz, CDCl ${ }_{3}$ ) $\delta 171.9$ (s, C-Ar), 167.5 (s, C(O)), 135.4 (s, C=), 134.4 (s, C-Ar), 133.6 (s, C-Ar), 128.1 (s, CH-Ar), 124.9 (s, CH-Ar), 123.8 (s, C-Ar), 122.9 (s, $\mathrm{CH}_{2}=$ ), 110.6 (s, CH-Ar), 60.1 (s, C-2), 27.1 (s, $\mathrm{CH}_{3} \mathrm{CH}_{2}$ ), 21.0 (s, CH $\mathrm{CH}_{3}$, $8.4\left(\mathrm{~s}, \mathrm{CH}_{3} \mathrm{CH}_{2}\right.$ ). Anal. Calcd for $\mathrm{C}_{14} \mathrm{H}_{14} \mathrm{~N}_{2} \mathrm{OS}$ (258.34): C, 65.09; $\mathrm{H}, 5.46 ; \mathrm{N}, 10.84 \%$. Found: $\mathrm{C}, 64.94 ; \mathrm{H}, 5.50 ; \mathrm{N}$, $10.87 \%$.

4-Isopropyl-8-methyl-3-methylene-3,4-dihydro-2H-benzo[4,5]thiazolo[3,2-a]pyrimidin-2-one (13h). (39\%); yellow oil; IR v(cm $\left.{ }^{-1}\right): 2958(\mathrm{w}), 1685$ (m), 1489 (vs), 1347 (s), $1172(\mathrm{~m}), 746(\mathrm{~m}) ;{ }^{1} \mathrm{H} \mathrm{NMR}\left(700 \mathrm{MHz}, \mathrm{CDCl}_{3}\right) \delta$ 7.38 (s, 1H, H-Ar), 7.22 (d, J $8.3 \mathrm{~Hz}, 1 \mathrm{H}, H-\mathrm{Ar}$ ), 7.01 (d, J $8.3 \mathrm{~Hz}, 1 \mathrm{H}, H-\mathrm{Ar}$ ), $6.46(\mathrm{~s}, 1 \mathrm{H}, \mathrm{CH}=\mathrm{C}), 5.48(\mathrm{~s}, 1 \mathrm{H}$, $\left.\mathrm{CH}_{2}=\mathrm{C}\right), 4.85(\mathrm{~d}, J 4.0 \mathrm{~Hz}, 1 \mathrm{H}, \mathrm{H}-4), 2.42\left(\mathrm{~s}, 3 \mathrm{H}, \mathrm{CH}_{3}\right), 2.37$ (heptd, J 6.9, 4.0, $\left.1 \mathrm{H}, \mathrm{CH}\left(\mathrm{CH}_{3}\right)_{2}\right), 1.02(\mathrm{~d}, J 6.9 \mathrm{~Hz}, 3 \mathrm{H}$, $\left.\mathrm{CH}_{3}\right), 0.86\left(\mathrm{~d}, J 6.9 \mathrm{~Hz}, 3 \mathrm{H}, \mathrm{CH}_{3}\right) ;{ }^{13} \mathrm{C}$ NMR $\left(176 \mathrm{MHz}, \mathrm{CDCl}_{3}\right) \delta 172.4$ (s, C-Ar), 168.2 (s, C(O)), 135.7 (s, C=), 134.4 (s, C-Ar), 131.2 (s, C-Ar), 128.0 (s, CH-Ar), 126.3 (s, CH-Ar), 123.9 (s, C-Ar), 123.0 (s, CH $=110.9$ (s, CH-Ar), 64.4 (s, C-2), 31.9 (s, $\mathrm{CH}_{3} \mathrm{CH}$ ), 21.1 (s, $\left.\mathrm{CH}_{3}\right), 18.1$ (s, $\mathrm{CH}_{3} \mathrm{CH}$ ), 15.9 (s, $\mathrm{CH}_{3} \mathrm{CH}$ ). Anal. Calcd for $\mathrm{C}_{15} \mathrm{H}_{16} \mathrm{~N}_{2} \mathrm{OS}$ (272.37): C, $66.15 ; H, 5.92 ; N, 10.29 \%$. Found: $C, 66.01 ; H, 5.93 ; N, 10.33 \%$. 
4-Butyl-8-methyl-3-methylene-3,4-dihydro-2H-benzo[4,5]thiazolo[3,2-a]pyrimidin-2-one (13i). (87\%); yellow oil; IR v(cm ${ }^{-1}$ ): 2954 (w), 1665 (m), 1484 (vs), 1352 (s), 1152 (m), 746 (m); ${ }^{1} \mathrm{H}$ NMR (700 MHz, CDCl $) \delta 7.33$ (s, $1 \mathrm{H}, H$-Ar), 7.20 (d, J $8.3 \mathrm{~Hz}, 1 \mathrm{H}, H-\mathrm{Ar}), 7.04$ (d, J $8.3 \mathrm{~Hz}, 1 \mathrm{H}, \mathrm{H}-\mathrm{Ar}), 6.33\left(\mathrm{~s}, 1 \mathrm{H}, \mathrm{CH}_{2}=\mathrm{C}\right), 5.50\left(\mathrm{~s}, 1 \mathrm{H}, \mathrm{CH}_{2}=\mathrm{C}\right), 4.99$ (dd, J 8.8, 3.8 Hz, 1H, H-4), $2.36\left(\mathrm{~s}, 3 \mathrm{H}, \mathrm{CH}_{3}\right), 1.83-1.77\left(\mathrm{~m}, 1 \mathrm{H}, \mathrm{CH}_{2}\right), 1.75-1.69\left(\mathrm{~m}, 1 \mathrm{H}, \mathrm{CH}_{2}\right), 1.30-1.15(\mathrm{~m}$, $4 \mathrm{H}, \mathrm{CH}_{2}$ ), 0.79 (t, J $\left.7.0 \mathrm{~Hz}, 3 \mathrm{H}, \mathrm{CH}_{3}\right) ;{ }^{13} \mathrm{C} \mathrm{NMR}\left(176 \mathrm{MHz}, \mathrm{CDCl}_{3}\right) \delta 171.5$ (s, C-Ar), 167.5 (s, C(O)), 135.3 (s, C=), 134.3 (s, C-Ar), 134.0 (s, C-Ar), 128.1 (s, CH-Ar), 124.7 (s, CH-Ar), 123.7 (s, C-Ar), 122.8 (s, CH $=$ ), 110.5 (s, CHAr), 59.0 (s, C-2), 33.5 (s, $\mathrm{CH}_{2}$ ), 25.7 (s, $\left.\mathrm{CH}_{2}\right), 22.0\left(\mathrm{~s}, \mathrm{CH}_{2}\right), 20.7$ (s, $\left.\mathrm{CH}_{3}\right), 13.6\left(\mathrm{~s}, \mathrm{CH}_{3}\right)$. Anal. Calcd for $\mathrm{C}_{16} \mathrm{H}_{18} \mathrm{~N}_{2} \mathrm{OS}$ (286.39): C, 67.10; H, 6.34; N, 9.78\%. Found: C, 67.01; H, 6.35; N, 9.76\%.

8-Methyl-3-methylene-4-phenyl-3,4-dihydro-2H-benzo[4,5]thiazolo[3,2-a]pyrimidin-2-one (13j). (71\%);

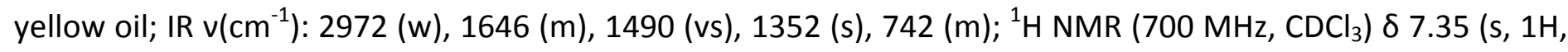
$H$-Ar), $7.33-7.28$ (m, 2H, H-Ar), $7.23-7.19$ (m, 1H, H-Ar), 7.06 (d, J 8.3 Hz, 1H, H-Ar), 6.85 (d, J $8.3 \mathrm{~Hz}, 1 \mathrm{H}, H$ $\mathrm{Ar}), 6.39\left(\mathrm{~s}, 1 \mathrm{H}, \mathrm{CH}_{2}=\mathrm{C}\right), 6.06\left(\mathrm{~s}, 1 \mathrm{H}, \mathrm{CH}_{2}=\mathrm{C}\right), 5.70(\mathrm{~s}, 1 \mathrm{H}, \mathrm{H}-4), 2.35\left(\mathrm{~s}, 3 \mathrm{H}, \mathrm{CH}_{3}\right) ;{ }^{13} \mathrm{C} \mathrm{NMR}\left(176 \mathrm{MHz}^{\mathrm{C}} \mathrm{CDCl}\right)_{3} \delta$ 172.7 (s, C-Ar), 166.6 (s, C(O)), 137.9 (s, C=), 135.8 (s, C-Ar), 134.8 (s, C-Ar), 134.6 (s, C-Ar), 129.6 (s, 2 x CH-Ar), 128.9 (s, CH-Ar), 128.1 (s, CH-Ar), 125.5 (s, CH-Ar), 125.4 (s, 2 x CH-Ar), 123.6 (s, C-Ar), 122.8 (s, CH $\mathrm{CH}-\mathrm{Ar}$ ), 62.7 (s, C-2). Anal. Calcd for $\mathrm{C}_{18} \mathrm{H}_{14} \mathrm{~N}_{2} \mathrm{OS}$ (306.38): C, 70.56; H, 4.61; N, 9.14\%. Found: C, 70.52; $\mathrm{H}, 4.62$; N, $9.15 \%$.

4-Butyl-8-methoxy-3-methylene-3,4-dihydro-2H-benzo[4,5]thiazolo[3,2-a]pyrimidin-2-one (13m). (76\%); yellow oil; IR v(cm $\left.{ }^{-1}\right): 2953$ (w), 1663 (m), 1481 (vs), 1355 (s), 1270 (s), 1150 (s), 808 (m); ${ }^{1} \mathrm{H} \mathrm{NMR}(700 \mathrm{MHz}$, $\left.\mathrm{CDCl}_{3}\right) \delta 7.09$ (d, J $\left.2.5 \mathrm{~Hz}, 1 \mathrm{H}, H-\mathrm{Ar}\right), 7.06$ (d, J $\left.8.9 \mathrm{~Hz}, 1 \mathrm{H}, H-\mathrm{Ar}\right), 6.98$ (dd, J 8.9, $\left.2.5 \mathrm{~Hz}, 1 \mathrm{H}, H-\mathrm{Ar}\right), 6.37(\mathrm{~s}, 1 \mathrm{H}$, $\left.\mathrm{CH}_{2}=\mathrm{C}\right), 5.50\left(\mathrm{~s}, 1 \mathrm{H}, \mathrm{CH}_{2}=\mathrm{C}\right), 4.97(\mathrm{dd}, J 8.8,3.8 \mathrm{~Hz}, 1 \mathrm{H}, \mathrm{H}-4), 3.82\left(\mathrm{~s}, 3 \mathrm{H}, \mathrm{CH}_{3} \mathrm{O}\right), 1.88-1.81\left(\mathrm{~m}, 1 \mathrm{H}, \mathrm{CH}_{2}\right), 1.78-$ $1.72\left(\mathrm{~m}, 1 \mathrm{H}, \mathrm{CH}_{2}\right), 1.33-1.19\left(\mathrm{~m}, 4 \mathrm{H}, \mathrm{CH}_{2}\right), 0.83\left(\mathrm{t}, J 7.1 \mathrm{~Hz}, 3 \mathrm{H}, \mathrm{CH}_{3}\right) ;{ }^{13} \mathrm{C} \mathrm{NMR}\left(176 \mathrm{MHz}, \mathrm{CDCl}_{3}\right) \delta 171.6(\mathrm{~s}, \mathrm{C}-$ Ar), 167.4 (s, C(O)), 156.8 (s, C-Ar), 134.0 (s, C=), 131.4 (s, C-Ar), 125.2 (s, CH-Ar), 124.8 (s, C-Ar), 114.3 (s, C-Ar), 111.6 (s, $\mathrm{CH}_{2}=$ ), 107.5 (s, CH-Ar), 59.3 (s, C-2), 55.9 (s, CH $\mathrm{CH}_{3} \mathrm{O}$ ), 33.6 (s, $\mathrm{CH}_{2}$ ), 25.8 (s, CH $\mathrm{CH}_{2}$, 22.1 (s, CH ), 13.7 (s, $\mathrm{CH}_{3}$ ). Anal. Calcd for $\mathrm{C}_{16} \mathrm{H}_{18} \mathrm{~N}_{2} \mathrm{O}_{2} \mathrm{~S}$ (302.39): C, 63.55; H, 6.00; N, 9.26\%. Found: C, 63.42; H, 6.04; N, 9.30\%.

\section{Acknowledgements}

This work was financially supported by the National Science Centre of Poland (project DEC2012/07/B/ST5/02006).

\section{References}

1. Vitaku, E.; Smith, D. T.; Njardarson, J. T. J. Med. Chem. 2014, 57, 10257-10274.

\section{http://dx.doi.org/10.1021/jm501100b}

2. Harutyunyan, A. A.; Panosyan, G. A.; Chishmarityan, S. G.; Tamazyan, R. A.; Aivazyan, A. G.; Paronikyan, R. V.; Stepanyan, H. M.; Sukasyan, R. S.; Grigoryan, A. S. Russ. J. Org. Chem. 2015, 51, 711-714. http://dx.doi.org/10.1134/S107042801505022X

3. Hilal, H. S.; Ali-Shtayeh, M. S.; Arafat, R.; Al.-Tel, T.; Voelter, W.; Barakat, A. Eur. J. Med. Chem. 2006, 41, 1017-1024.

\section{http://dx.doi.org/10.1016/i.ejmech.2006.03.025}

4. Kumar, G.; Sharma, P. K.; Sharma, S.; Singh, S. J. Chem. Pharm. Res. 2015, 7, 710-714.

5. Richardson, A.; McCarty, F. J. J. Med. Chem. 1972, 15, 1203-1206.

\section{http://dx.doi.org/10.1021/jm00282a001}


6. Wade, J. J.; Toso, C. B.; Matson, Ch. J.; Stelzer, V. L. J. Med. Chem. 1983, 26, 608-611. http://dx.doi.org/10.1021/jm00358a031

7. Bartovic, A.; llavsky, D.; Simo, O.; Zalibera, L.; Belicova, A.; Seman, M. Collect. Czech. Chem. Commun. 1995, 60, 583-593. http://dx.doi.org/10.1135/cccc19950583

8. Ahmad, N. M.; Jones, K. Tetrahedron Lett. 2010, 3264-3265.

9. Prasad, P. R.; Shinde, S. D.; Waghmare, G. S.; Naik, V. L.; Bhuvaneswari, K.; Kuberkar, S. V. J. Chem. Pharm. Res. 2011, 3, 20-27.

10. Prasad, P. R.; Bhuvaneswari, K.; Kumar, K. P.; Rajani, K.; Kuberkar, S. V. J. Chem. Pharm. Res. 2012, 4, 1606-1611.

11. Refat, H. M.; Fadda, A. A. Heterocycles 2015, 91, 1212-1226.

http://dx.doi.org/10.3987/COM-15-13209

12. Chadegani, C.; Darviche, D.; Balalaie, B. Int. J. Org. Chem. 2012, 2, 31-37.

http://dx.doi.org/10.4236/ijoc.2012.21006

13. Wahe, H.; Mbafor, J. T.; Nkengfack, A. E.; Fomum, Z. T.; Cherkasov, R. A.; Sterner, O.; Doepp, D. Arkivoc, 2003, xv, 107-114.

14. Gabr, M. T.; El-Gohary, N. S.; El-Bendary, E. R.; El-Kerdawy, M. M. Med. Chem. Res. 2015, 24, 860-878. http://dx.doi.org/10.1007/s00044-014-1114-x

15. Gabr, M. T.; El-Gohary, N. S.; El-Bendary, E. R.; El-Kerdawy, M. M. Eur. J. Med. Chem. 2014, 85, 576-592. http://dx.doi.org/10.1016/j.ejmech.2014.07.097

16. Eriskin, S.; Sener, N.; Yavuz, S.; Sener, I. Med. Chem. Res. 2014, 23, 3733-3743. http://dx.doi.org/10.1007/s00044-014-0962-8

17. Satyanarayana, S.; Kumar, K. P.; Reddy, P. L.; Narender, R.; Narasimhulu, G. Tetrahedron Lett. 2013, 54, 4892-4895.

http://dx.doi.org/10.1016/j.tetlet.2013.06.138

18. Kitson, R. R. A.; Millemaggi, A.; Taylor, R. J. K. Angew. Chem. Int. Ed. 2009, 48, 9426-9452. http://dx.doi.org/10.1002/anie.200903108

19. Albrecht, A.; Albrecht, Ł.; Janecki, T. Eur. J. Org. Chem. 2011, 15, 2747-2766.

http://dx.doi.org/10.1002/ejoc.201001486

20. Pięta, M.; Kędzia, J.; Janecka, A.; Pomorska, D. K.; Różalski, M.; Krajewska, U.; Janecki, T. RSC Adv. 2015, 5, 78324-78335 and references cited therein.

http://dx.doi.org/10.1039/C5RA16673J

21. Modranka, J.; Janecki, T. Tetrahedron 2011, 67, 9595-9601.

http://dx.doi.org/10.1016/j.tet.2011.09.139

22. Pietrzak A., Modranka J., Wojciechowski J., Janecki T.; Wolf W. M., The Cambridge Structural Database, CCDC 1478184, 2016.

23. Smith, M. B.; In March`s Advanced Organic Chemistry, $7^{\text {th }}$ Edition; John Wiley \& Sons, Inc., Hoboken, New Jersey, 2013; p. 867.

24. Janecki, T.; Wąsek, T.; Różalski, M.; Krajewska, U.; Studzian, K.; Janecka, A. Bioorg. Med. Chem. Lett. 2006, 16, 1430-1433.

http://dx.doi.org/10.1016/j.bmcl.2005.11.032

25. Janecki, T.; Wąsek, T. Tetrahedron 2004, 60, 1049-1055.

http://dx.doi.org/10.1016/j.tet.2003.11.083 
26. Modranka, J.; Albrecht, A.; Janecki, T. Synlett 2010, 2867-2870.

27. Groom C. R.; Bruno I. J.; Lightfoot M. P.; Ward S. C. Acta Cryst. 2016. B72, 171-179.

28. Bruker (2014). APEX2. Bruker-Nonius AXS Inc., Madison, Wisconsin, USA. 